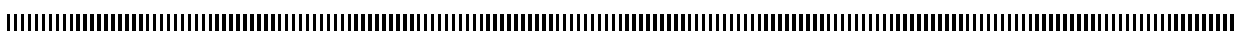
|

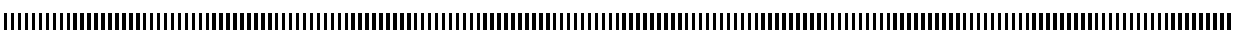
| | | |

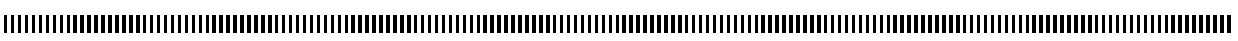

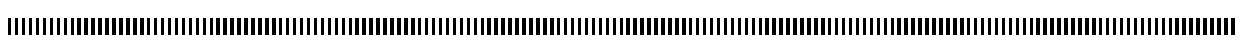

\title{
A discrete free boundary problem
}

\author{
Imme van den Berg \\ Departamento de Matemática \\ Universidade de Évora \\ Évora \\ Portugal \\ ivdb@uevora.pt
}

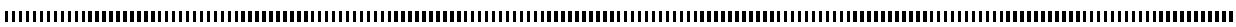

ABSTRACT. We study the free boundary problem in a nonstandard setting of infinitesimal discretisations of the heat equation. In particular we derive regularity results of solutions and the free boundary, in terms of S-continuity and S-differentiability.

RÉSUMÉ. Nous étudions le problème de la frontière libre dans le contexte nonstandard des discrétisations infinitésimales de l'équation de la chaleur. En particulier nous prouvons des résultats de régularité des solutions et de la frontière libre, en termes de S-continuité et de S-dérivabilité.

KEYWORDS : Free boundary problem, discrete heat equation, S-continuity, S-differentiability, nonstandard analysis.

MOTS-CLÉS : Problème de frontière libre, équation de la chaleur discrète, S-continuité, S-dérivabilité, analyse nonstandard.

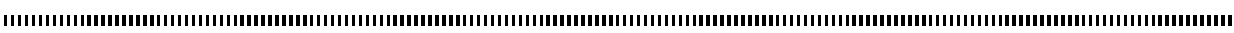




\section{Contents}

1 Introduction 2

2 The discrete free boundary problem 5

3 Tools from probability 6

3.1 The Wiener walk and the binomial distribution . . . . . . . . . . 6

3.2 Path sums and the Principle of Reflection $\ldots \ldots \ldots \ldots$

4 Solutions and upper solutions $\quad 10$

4.1 Solutions and upper solutions as path sums $\ldots \ldots \ldots \ldots$

4.2 Differences in space and time . . . . . . . . . . . . . . . . . 12

5 Continuous approximations of discrete formulas 13

5.1 Regularity of the binomial function . . . . . . . . . . . . . . . . . 14

5.2 Estimates of probabilities of transition . . . . . . . . . . . . 16

5.3 Estimates of path sums $\ldots \ldots \ldots \ldots \ldots$

6 Hypotheses, some examples, earlier approaches 20

$7 \quad S$-continuity of the free boundary and the solution 25

$7.1 S$-continuity of the free boundary . . . . . . . . . . . . . . . 25

$7.2 S$-continuity of the solution in space . . . . . . . . . . . 27

$7.3 S$-continuity of the solution in time . . . . . . . . . . . 28

8 Higher order $S$-derivability of the solutions 29

$9 S$-derivability of the solution infinitely close to the free boundary 32

$9.1 S$-derivability of the solution in space . . . . . . . . . . . . 32

$9.2 S$-derivability of the solution in time . . . . . . . . . . . . . . 34

9.3 Non $S$-derivability in $2^{\text {nd }}$ order of the solution in space $\ldots \ldots \ldots . \ldots 36$

10 References $\quad 36$

\section{Introduction}

Consider the backward heat equation on some domain, with a given condition at the horizon. Assume a second condition is prescribed, in the form of a fixed function on the domain. The free boundary problem consists in determining the subdomain where the prescribed function is maximal, and the subdomain where the solution of the heat equation is maximal, together with the values of the solution. Under appropriate conditions the two 
subdomains, called stopping region and continuation region, are separated by a simple curve, which is a priori unknown. This curve is called the free boundary, and thus must be determined simultaneously with the solution.

Such free boundary problems occur in several settings. One of them is the so-called Stefan-problem: in a frozen lake, part of the water is heated, and one should determine the curve separating the ice and the melting region. Another example came up with financial mathematics, and deals with the American put option, which gives the owner the right to sell a stock anytime at a previously fixed price, see for instance [9]. Then the stopping region is the set of values of the stock where it is optimal to sell, and the set of values where it optimal to stay with the stock is the continuation region. This free boundary problem deals with stochastics, but after some appropriate adaptations it may be shown to be equivalent to the setting in terms of the heat equation. Then in the continuation region the process behaves as a martingale (constant conditional expectations), while in the stopping region the process has decreasing conditional expectations. Then as a whole the process is a supermartingale, and the free-boundary problem may be reformulated as to determine the smallest supermartingale satisfying all conditions, the so-called Snellenvelope.

The free boundary problem is still largely open. Progress has been made in proving properties of regularity (continuity, differentiability) of the solution and the free boundary, using rather strong techniques, like analytic functions and series (Gevrey [8])), functional analysis (Friedman [7]) and stochastic integration (Van Moerbeke [11][12]). Mostly some a priori conditions are assumed, such as the continuation region and the stopping region being separated by a curve, or a finite family of such curves.

The present article is written in the setting of discretisations of the heat equation. We take as a starting point a one-step dynamic optimization problem, which gives rise to the discrete heat equation by taking to two time-steps. We consider the case where the free boundary has the form of a "discrete curve". Then in the continuation region the solution has the form of a finite sum. We work on an infinitesimal grid in the sense of nonstandard analysis. The article aims to obtain nonstandard regularity properties of this solution and the free boundary, formulated in terms of $S$-continuity and $S$-differentiability by elementary means, in the spirit of [13] and [2]; for an introduction to the axiomatic form of nonstandard analysis $I S T$ and terminology used here, we refer to [6].

We assume that the condition at the horizon is $S$-differentiable once, but not twice, otherwise the prescribed function is two times $S$-differentiable in space and once in time, with $S$-continuous discrete partial derivatives, all satisfy some growth condition. We make the rather strong a priori assumption that the free boundary has a property of monotony. Then the results on regularity are resumed as follows. The free boundary is at least locally $S$-continuous, the solution has $S$-continuous discrete partial derivatives of all standard order at points of the continuation region not infinitely close to the free boundary or the horizon, and infinitely close to the free boundary discrete partial derivatives of first order are $S$-continuous, but not the discrete partial derivative of second order with respect 
to space. The regularity properties of the solution proved here are about as strong as those proved by Friedman and Van Moerbeke, but as regards to the regularity properties of the free boundary, they also show that it is differentiable.

We observe that in the context of the heat equation the interaction between the continuous and the discrete approach is not as obvious as in the case of ordinary difference equations-ordinary differential equations, because differentiability is not always verified. For instance, normally at the horizon the continuous solution is not two-times differentiable in space. So one has to refer to more indirect methods of functional analysis, or alternatively one has to relate finite-time stochastics and the much more involved continuous-time stochastics. A nonstandard article which deals with the transition between the discrete and the continuous in the context of the Snell-envelope for the American put is given by Cutland, Kopp, Willinger and Wyman [5]. Using Loeb-measure, they prove a convergence property ( $D^{2}$-convergence) for the solution of the discrete free boundary problem, which is stronger than weak convergence. In [10] classical convergence results on discrete approximations of the continuous free-boundary problem are presented, and a bibliography.

This article has the following structure. We start by formulating the discrete free boundary problem in Section 2, and by stating some basic properties of the discrete heat equation and its solutions in Section 3. Subsection 3.1 relates the discrete heat equation with its stochastic solution in the form of an expectation, where we will use a sort of semi-continuous notation. In Subsection 3.2 expectations are written in convenient form, so-called path sums, which are the finite analogue of path integrals. Within our semicontinuous notation we develop some tools in order to deal with the path sums, based on the Reflection principle. Applying path sums to the free boundary problem, Subsection 4 gives concrete expressions for the solution, and also useful forms for upper and lower solutions. The latter yield bounds of differences of solutions, which enable proofs of the regularity properties; the approach by upper and lower solutions is not very different form the methods used in [11].

In Section 5 continuous approximations are made of discrete formulas, taking advantage of the semi-continuous notation. In particular it recalls the DeMoivre-Laplace Theorem of [4], which extends the approximation of the binomial distribution by the normal distribution to all standard difference quotients and differential quotients. Also some useful estimates of path-sums are given in terms of improper Riemann-integrals.

In Section 6 we formulate the regularity properties of the prescribed function and the condition at the horizon needed in the remaining part of the article. We illustrate the free boundary problem with some simple examples which may treated by direct calculations. We compare the conditions and results on regularity with those of the articles by Van Moerbeke and Friedman.

The properties of $S$-continuity and $S$-differentiability are progressively proved in Section 7,8 and 9 . 


\section{The discrete free boundary problem}

Let $\delta t>0$ be a fixed time-step and $\mathbb{T}=\mathbb{N} \delta t$. Let $T \in \mathbb{T}, T>0$; for convenience we suppose that $T$ is standard and that $T / \delta t$ is even. Let $\delta x=2 \sqrt{\delta t}$ be the space-step, and let $\mathbb{X}=\mathbb{Z} \delta x$. We consider the grid composed of points $(t, x)$ for $t / \delta t$ even, and $(t, x+\sqrt{\delta t})$ for $t / \delta t$ odd, where $t \in \mathbb{T}$ and $x \in \mathbb{X}$. We let $D$ be the grid restricted to $0 \leq t \leq T$, then $T$ is called the horizon. Let $g: D \rightarrow \mathbb{R}$. In this setting the free boundary problem is given by

$$
\left\{\begin{array}{l}
u(t, x)=\max (\mu(t, x), g(t, x)) \\
u(T, x)=g(T, x)
\end{array}\right.
$$

where

$$
\mu(t, x)=\frac{1}{2} u(t+\delta t, x+\sqrt{\delta t})+\frac{1}{2} u(t+\delta t, x-\sqrt{\delta t}) .
$$

Often it is convenient to write the condition at the horizon $T$ separately by $g(T, x) \equiv$ $h(x)$. The stopping region $S$ is the set of all points $(t, x) \in D$ such that $u(t, x)$ has the fixed value $g(t, x)$; we suppose for convenience that the stopping region includes the points where $\mu(t, x)=g(t, x)$. In the continuation region $C$ the value $u(t, x)$ is given by the mean $\mu(t, x)$. The free boundary separates $C$ and $D$; it may or may not be a simple (discrete) curve. The search for the solution of (1) involves the simultaneous search for the free boundary.

Observe that the solution $u$ satisfies in the continuation region the martingale property: at time $t$ the value of the solution $u(t, \cdot)$ is the mean (2) of the solution $u(t+\delta t, \cdot)$ at the next time $t+\delta t$. In the stopping region

$$
g(t, x) \geq \frac{1}{2} g(t+\delta t, x+\sqrt{\delta t})+\frac{1}{2} g(t+\delta t, x-\sqrt{\delta t}) .
$$

As a consequence, the solution of the free boundary problem (1) has the supermartingale property

$$
u(t, x) \geq \frac{1}{2} u(t+\delta t, x+\sqrt{\delta t})+\frac{1}{2} u(t+\delta t, x-\sqrt{\delta t}) .
$$

To see the relation with the discrete heat equation, we denote the difference quotient in time by

$$
u_{1,0}(t, x)=\frac{u(t+2 \delta t, x)-u(t, x)}{2 \delta t} .
$$

Note that due to the construction of the grid $D$ differences in time of the function $u$ imply an even number of time-steps. The first-order difference quotient in space is denoted by

$$
u_{0,1}(t, x)=\frac{u(t, x+\delta x)-u(t, x)}{\delta x},
$$

In general $u_{m, n}$ denotes the $m^{t h}$-order difference quotient in time of the $n^{t h}$-order difference quotient in space of $u$. 
Then taking two successive steps of the backward recurrence relation

$$
u(t, x)=\frac{1}{2} u(t+\delta t, x+\sqrt{\delta t})+\frac{1}{2} u(t+\delta t, x-\sqrt{\delta t}),
$$

one shows that the function $u$ satisfies the backward discrete heat equation

$$
u_{1,0}(t-2 \delta t, x)+\frac{1}{2} u_{0,2}(t, x-\delta x)=0,
$$

as long as we remain in $C$.

We did not take this equation as a starting point, for taking time-steps $2 \delta t$ may imply that $u$ is not defined on the whole of $D$.

We assume from now on that the continuation region and the stopping region are separated by one discrete curve $\phi$. To fix ideas we suppose that $C$ lies on top of $S$, this is no essential restriction. We also assume that the curve $\phi$ lies within the stopping region and that $\phi$ is a function of time. Then $\phi(t)$ satisfies for $t \in[0 . . T]$

$$
\phi(t)=\max \{x \mid(t, x) \in S\} .
$$

Observe that by supposing $\phi$ functional it is avoided that the boundary has "horizontal parts", in the sense that two points $(t, x),(t, x+\delta x) \in D$, with $(t-\delta t, x+\sqrt{\delta t}) \notin D$. This property is satisfied if (3) is supposed to hold everywhere. Section 6 will present some concrete examples where these conditions are satisfied (it may happen that $\phi$ is defined only on some discrete interval $\left[t^{\prime} . . T\right]$ with $0<t^{\prime} \leq T$, but it is always possible to consider the free boundary problem on a restricted time-interval).

\section{Tools from probability}

\subsection{The Wiener walk and the binomial distribution}

As is well-known, the solution of equation (4) is an expectation with respect to the Wiener walk. The Wiener walk $W$ is the discrete stochastic process (sequence of random variables $W_{t}$ ) with independent increments $\delta W_{t} \equiv W_{t+\delta t}-W_{t}$, defined by $W_{0}=0$ and

$$
\delta W_{t}=\left\{\begin{array}{cl}
\sqrt{\delta t} & \text { probability } \frac{1}{2} \\
-\sqrt{\delta t} & \text { probability } \frac{1}{2},
\end{array}\right.
$$

where $0 \leq t<T$. We write $\Lambda_{t, x}$ the set of all (equiprobable) trajectories starting at $(t, x)$. We denote by $\Gamma$ the set of all points on at least one trajectory of $\Lambda_{0,0}$. By independence, the probability $p r$ of every trajectory $\lambda \in \Lambda_{t, x}$ satisfies

$$
\operatorname{pr}(\lambda)=\left(\frac{1}{2}\right)^{(T-t) / \delta t} .
$$


Then $\left\langle\Lambda_{t, x}, p r>\right.$ is a probability space. If we consider the equation (4) only with a condition $h(x)$ at the horizon, one shows with backward induction that its solution is given by the expectation

$$
u(t, x)=\sum_{\lambda \in \Lambda_{t, x}} h(\lambda(T)) \operatorname{pr} \lambda .
$$

Grouping trajectories with the same endpoint yields a second manner to express $u(t, x)$. This gives way to the binomial distribution, given by

$$
B(\nu, j)=\left(\begin{array}{l}
\nu \\
j
\end{array}\right)\left(\frac{1}{2}\right)^{\nu}
$$

where $\nu, j \in \mathbb{N}$ are such that $0 \leq j \leq \nu$. We do not write the expectations in terms of the binomial coefficients, instead we use the scaling of [3].

Definition 1. Let $(t, x) \in \Gamma$. We write

$$
\begin{aligned}
\nu_{t} & =\frac{t}{\delta t} \\
j_{t, x} & =\frac{t}{2 \delta t}+\frac{x}{\delta x} .
\end{aligned}
$$

Definition 2. The binomial function $b: \Gamma \rightarrow \mathbb{R}$ is defined by

$$
b(t, x)=\frac{1}{\delta x} B\left(\nu_{t}, j_{t, x}\right)
$$

The substitutions of Definition 1 may be interpreted by a centralization of the binomial distribution around the mean $\nu / 2$ and a reduction of the distance to the mean by

$$
\delta x=2 \sqrt{\delta t}=\frac{2}{\sqrt{\nu}},
$$

which is equal to the standard deviation when $t=1$. The centralization, reduction and blow-up of the binomial coefficients with the factor $1 / \delta x$ permit to formulate a version of the DeMoivre-Laplace Central Limit Theorem, which does not involve a change of variables, see Section 5.

The following formulas for recurrences in space of the binomial function are obtained by straightforward calculation (see $[3, \mathrm{p} .17,18]$ ).

Lemma 1. Let $(t, x) \in \Gamma$ with $t>0$. Then

$$
b(t, x+\delta x)=b(t, x) \frac{1-\frac{x}{2 t} \delta x}{1+\frac{x}{2 t} \delta x+\frac{2}{t} \delta t}
$$

and

$$
b(t-\delta t, x+\sqrt{\delta t}) \delta x-b(t-\delta t, x-\sqrt{\delta t}) \delta x=-4 b(t-\delta t, x-\sqrt{\delta t}) \frac{x}{t+x \sqrt{\delta t}} \delta t .
$$




\subsection{Path sums and the Principle of Reflection}

Expectations on the grid $D$ may be written in the form of "path sums", which are sums over trajectories of the Wiener walk. These path sums are analogues of path integrals, but are of course easier to define in the present finite setting. The counting of paths is less obvious, with the notable exception of Andrés Principle of Reflection (1875). Below we recall this principle, using the coordinates of the grid $D$ and the binomial function.

Definition 3. Let $T \in \mathbb{T}$. A function $\phi:[0 \cdot T] \rightarrow \mathbb{Z} \sqrt{\delta t}$ is called of minimal variation if for all $t<T$

$$
\phi(t+\delta t)=\phi(t)+\sqrt{\delta t} \vee \phi(t+\delta t)=\phi(t)-\sqrt{\delta t} .
$$

It is called weakly nondecreasing if $\phi\left(t^{\prime}\right) \geq \phi(t)$ for all $t<t^{\prime} \leq T$ such that $\left(t^{\prime}-t\right) / \sqrt{\delta t}$ is even.

Trajectories of the Wiener walk are of minimal variation. A weakly nondecreasing function of minimal variation allows for downward steps $-\sqrt{\delta t}$, but they never may be followed by a second downward step. An example of a nondecreasing function of minimal variation is the "horizontal" trajectory given by $\phi(t)=-\sqrt{\delta t}+(-1)^{t / \delta t} \sqrt{\delta t}$.

Differentiable standard functions give rise to functions of minimal variation, if the discretisations are as follows.

Assume $T>0$ is standard and let $f:[0, T] \rightarrow \mathbb{R}$ be standard and differentiable. Define $\phi:[0 \cdots T] \rightarrow \mathbb{Z} \sqrt{\delta t}$ such that $(t, \phi(t)) \in D, \phi(t) \leq f(t)$ and $f(t)-\phi(t)$ is minimal for all $t \in[0 \cdot T]$. Since $|f(t+\delta t)-f(t)| \leq K \delta t$ for some standard $K \geq 0$, one can only have $|\phi(t+\delta t)-\phi(t)| \leq \sqrt{\delta t}$. Observe that $\phi$ is weakly-nondecreasing if $f$ is nondecreasing; conversely the shadow of a weakly-nondecreasing function $\phi$ : $[0 \cdot T] \rightarrow \mathbb{Z} \sqrt{\delta t}$ such that $(t, \phi(t)) \in D$ for all $t \in[0 \cdot T]$ is nondecreasing (if it is a well-defined function).

Definition 4. Let $\phi:[0 \cdot T] \rightarrow \mathbb{Z} \sqrt{\delta t}$ be of minimal variation. Let $(t, x),(\tau, \xi) \in D$ with $t \leq \tau, x>\phi(t)$ and $\xi \geq \phi(\tau)$. The probability of transition from $(t, x)$ to $(\tau, \xi)$ is defined by

$$
P(t, x ; \tau, \xi)=\frac{\sharp\left\{\lambda \in \Lambda_{t, x} \mid \forall s(0 \leq s \leq \tau \Rightarrow \lambda(s)>\phi(s)) \wedge \lambda(\tau)=\xi\right\}}{\sharp \Lambda_{t, x}} ;
$$

trivially we define $P(t, \phi(t) ; t, \phi(t))=1$. In some cases, when the function $\phi$ is not clear from the context, we may write $P=P_{\phi}$.

Observe that we always have

$$
P(t, x ; \tau, \xi)=\frac{1}{2} P(t+\delta t, x+\sqrt{\delta t} ; \tau, \xi)+\frac{1}{2} P(t+\delta t, x-\sqrt{\delta t} ; \tau, \xi),
$$

for every trajectory going from $(t, x)$ to $(\tau, \xi)$ passes either through $(t+\delta t, x+\sqrt{\delta t})$ or through $(t+\delta t, x-\sqrt{\delta t})$, while the probability of the first trajectories is half of the probability of the latter two. 
In general there is no closed form formula for probabilities of transition. However the Principle of Reflection gives an interesting exception in case $\phi$ is "horizontal".

Proposition 2. Let $\lambda:[0 \cdot T] \rightarrow \mathbb{Z} \sqrt{\delta t}$ be defined by $\lambda(t)=-\sqrt{\delta t}+(-1)^{t / \delta t} \sqrt{\delta t}$. Let $(0, x),(t, y) \in D$ with $t, x, y>0$. Then

$$
P(0, x ; t, y)=(b(t, y-x)-b(t, y+x)) \delta x .
$$

Proof. We determine $b(t, y-x)-P(0, x ; t, y)$. Note that $b(t, y-x)$ represents the unrestricted probability of going from $(0, x)$ to $(t, y)$, which is equal to the probability of going from $(0,0)$ to $(t, y-x)$. Then $b(t, y-x)-P(0, x ; t, y)$ represents the probability of going from $(0, x)$ to $(t, y)$ with at least one hit of the boundary $\lambda$; observe that a first hit can only occur at a point where $\lambda$ takes the value 0 . Let

$$
\left\{\begin{array}{l}
A=\left\{\alpha \in \Lambda_{0, x} \mid \alpha(0)=x, \alpha(t)=y, \exists s(0<s<t \wedge \alpha(s)=0)\right\} \\
B=\left\{\beta \in \Lambda_{0, x} \mid \beta(0)=x, \beta(t)=-y\right\} .
\end{array}\right.
$$

Then $\sharp A=\sharp B$ by the following mapping $F$, which "reflects" a trajectory after its first hit of the boundary:

$$
F(\alpha)(s)= \begin{cases}\alpha(s) & \forall \tau(0 \leq \tau \leq s \Rightarrow \alpha(\tau)>0) \\ -\alpha(s) & \exists \tau(0 \leq \tau \leq s \wedge \alpha(\tau)=0) .\end{cases}
$$

Hence $\operatorname{Pr} A=\operatorname{Pr} B=b(t, x+y) \delta x$. We conclude that

$$
P(0, x ; t, y)=b(t, y-x) \delta x-\operatorname{Pr} A=(b(t, y-x)-b(t, y+x)) \delta x .
$$

Next proposition states the probability for a first hit of the boundary at time $t$.

Proposition 3. Let $\lambda:[0 \cdot T] \rightarrow \mathbb{R}$ be defined by $\lambda(t)=-\sqrt{\delta t}+(-1)^{t / \delta t} \sqrt{\delta t}$. Let $(0, x),(t, 0) \in D$ with $t, x>0$. Then

$$
P(0, x ; t, 0)=b(t-\delta t, x-\sqrt{\delta t}) \frac{x}{t+x \sqrt{\delta t}} 2 \delta t .
$$

Proof. Applying Lemma 1 we find

$$
\begin{aligned}
P(0, x ; t, 0) & =\frac{1}{2} P(0, x ; t-\delta t, \sqrt{\delta t}) \\
& =\frac{1}{2}(b(t-\delta t, x-\sqrt{\delta t}) \delta x-b(t-\delta t, x+\sqrt{\delta t}) \delta x) \\
& =b(t-\delta t, x-\sqrt{\delta t}) \frac{x}{t+x \sqrt{\delta t}} 2 \delta t
\end{aligned}
$$




\section{Solutions and upper solutions}

We present explicit formulas for solutions and upper solutions for the free boundary problem in terms of path sums. We use these formulas to express differences in time and space, and also lower and upper bounds of them.

We start by the equation (4) with final condition $h: \mathbb{X} \rightarrow \mathbb{R}$ given, still without prescribed function $g$. We rewrite the solution in the form of a Riemann sum. Remark that all values $\lambda(T)$ at the horizon of a trajectory $\lambda$ of the Wiener walk starting at $(t, x)$ satisfy $x-(T-t) / \sqrt{\delta t} \leq \lambda(T) \leq x+(T-t) / \sqrt{\delta t}$. Putting $\lambda(T)=x+y$, the probability to hit the horizon at $x+y$ is then given by $B\left(\nu_{T-t}, j_{t, y}\right)=b(T-t, y) \delta x$. Hence the expectation (6) becomes

$$
u(t, x)=\sum_{|y| \leq(T-t) / \sqrt{\delta t}} b(T-t, y) h(x+y) \delta x .
$$

\subsection{Solutions and upper solutions as path sums}

Let $\psi:[0 \cdots T] \rightarrow \mathbb{Z} \sqrt{\delta t}$ be a function of minimal variation. Let $(t, x) \in D$ with $x \geq \psi(t)$ and $t^{\prime} \leq T$. Let $\lambda$ be a trajectory of the Wiener walk starting at $(t, x)$. Then either it will hit the curve $\psi$ or it will hit the discrete line $t=t^{\prime}$ at some point $\left(t^{\prime}, y\right)$ with $\psi\left(t^{\prime}\right)<y \leq x+\left(t^{\prime}-t\right) / \sqrt{\delta t}$. Assume the value taken along the curve $\psi$ is given by some real valued function $\alpha$ and the value along $t=t^{\prime}$ by some function $\beta$.

We define $S_{\psi}(t, x ; \alpha)$ and $S_{t^{\prime}}(t, x ; \beta)$ by

$$
\begin{aligned}
& S_{\psi}(t, x ; \alpha)=\sum_{t \leq \tau \leq t^{\prime}} P(t, x ; \tau, \psi(\tau)) a(\tau, \psi(\tau)) \\
& S_{t^{\prime}}(t, x ; \beta)=\sum_{\psi\left(t^{\prime}\right)<y \leq x+\frac{t^{\prime}-t}{\sqrt{\delta t}}} P\left(t, x ; t^{\prime}, y\right) \beta\left(t^{\prime}, y\right) .
\end{aligned}
$$

Observe that it follows from (9) that for $t<t^{\prime}$ and $x>\psi(t)$

$$
\begin{aligned}
& S_{\psi}(t, x ; \alpha)=\frac{1}{2} S_{\psi}(t+\delta t, x+\sqrt{\delta t} ; \alpha)+\frac{1}{2} S_{\psi}(t+\delta t, x-\sqrt{\delta t} ; \alpha) \\
& S_{t^{\prime}}(t, x ; \beta)=\frac{1}{2} S_{t^{\prime}}(t+\delta t, x+\sqrt{\delta t} ; \beta)+\frac{1}{2} S_{t^{\prime}}(t+\delta t, x-\sqrt{\delta t} ; \beta) .
\end{aligned}
$$

If there is no ambiguity with respect to $(t, x)$ we simply write $S_{\psi}(t, x ; \alpha)=S_{\psi}(\alpha)$ and $S_{t^{\prime}}(t, x ; \beta)=S_{t^{\prime}}(\beta)$. With this notation the solution of (4) becomes

$$
u(t, x)=S_{\psi}(u)+S_{T}(g) .
$$


We may generalize this formula to express solutions of (1) with free boundary $\phi$, in case $\psi(t) \geq \phi(t)$ for $t \in\left[0 \cdot t^{\prime}\right]$ with $t^{\prime} \leq T$, i.e. we may stop before the horizon. Since the values at time $t^{\prime}$ have the form $u\left(t^{\prime}, y\right)$, formula (12) becomes

$$
u(t, x)=S_{\psi}(u)+S_{t^{\prime}}(u) .
$$

In the particular case where $\psi$ is equal to the free boundary $\phi$ we find

$$
u(t, x)=S_{\phi}(g)+S_{t^{\prime}}(u),
$$

and if also $t^{\prime}=T$ we may write, with $h(y)=g(T, y)$

$$
u(t, x)=S_{\phi}(g)+S_{T}(g)=S_{\phi}(g)+S_{T}(h) .
$$

If $\phi(\tau)<\psi(\tau)$ with $t \leq \tau \leq t^{\prime}$ one has $g(\tau, \psi(\tau)<u(\tau, \psi(\tau)$. Hence if $\phi(\tau)<$ $\psi(\tau)$ for all $\tau$ with $t \leq \tau \leq t^{\prime}$ it holds that

$$
u(t, x) \geq S_{\psi}(g)+S_{t^{\prime}}(u) .
$$

which represents an upper solution. We have also an upper solution if $\psi$ lies in the stopping region.

Theorem 4. Let $u$ satisfy the free boundary problem (1). Assume that $\psi:[0 \cdot T] \rightarrow \mathbb{Z} \sqrt{\delta t}$ and the free boundary $\phi:[0 \cdot T] \rightarrow \mathbb{Z} \sqrt{\delta t}$ are functions of minimal variation. Let $(t, x) \in$ $D$ with $x \geq \psi(t)$ and $t \leq t^{\prime} \leq T$. Assume that $\phi(\tau) \geq \psi(\tau)$ for all $\tau$ with $t \leq \tau \leq t^{\prime}$. Then

$$
u(t, x) \geq S_{\psi}(g)+S_{t^{\prime}}(u) .
$$

Proof. We apply backward induction in time. The formula holds with equality for $t=t^{\prime}$. Indeed, if $x>\phi\left(t^{\prime}\right)$, one has $u\left(t^{\prime}, x\right)=S_{t^{\prime}}(u)$ and $S_{\psi}(g)=0$, hence $u\left(t^{\prime}, x\right)=S_{\psi}(g)+$ $S_{t^{\prime}}(u)$. If $x \leq \phi\left(t^{\prime}\right)$ one has $u\left(t^{\prime}, x\right)=g\left(t^{\prime}, x\right)$. Then for $x>\psi\left(t^{\prime}\right)$ one has again $S_{\psi}(u)=0$ and $u\left(t^{\prime}, x\right)=S_{\psi}(u)+S_{t^{\prime}}(u)$, and for $x=\psi\left(t^{\prime}\right)$ one has $S_{t^{\prime}}(u)=0$ and $u\left(t^{\prime}, x\right)=g\left(t^{\prime}, \psi\left(t^{\prime}\right)\right)=S_{\psi}(g)=S_{\psi}(g)+S_{t^{\prime}}(u)$. Assume (17) holds at $t+\delta t$. Let $x>\phi(t)$. Using (11) we find

$$
\begin{aligned}
u(t, x)= & \mu(t, x)=\frac{1}{2} u(t+\delta t, x+\sqrt{\delta t})+\frac{1}{2} u(t+\delta t, x-\sqrt{\delta t}) \\
\geq & \frac{1}{2}\left(S_{\psi}(t+\delta t, x+\sqrt{\delta t} ; g)+S_{t^{\prime}}(t+\delta t, x+\sqrt{\delta t} ; u)\right)+ \\
& \frac{1}{2}\left(S_{\psi}(t+\delta t, x-\sqrt{\delta t} ; g)+S_{t^{\prime}}(t+\delta t, x-\sqrt{\delta t} ; u)\right) \\
= & \frac{1}{2}\left(S_{\psi}(t+\delta t, x+\sqrt{\delta t} ; g)+\frac{1}{2}\left(S_{\psi}(t+\delta t, x-\sqrt{\delta t} ; g)+\right.\right. \\
& \left.\frac{1}{2} S_{t^{\prime}}(t+\delta t, x+\sqrt{\delta t} ; u)+\frac{1}{2} S_{t^{\prime}}(t+\delta t, x+\sqrt{\delta t} ; u)\right) \\
= & S_{\psi}(u)+S_{t^{\prime}}(u) .
\end{aligned}
$$


If $x=\psi(t)$ we have $P(t, x ; t, \psi(t))=1$ and all other probabilities of transition are zero; so (17) holds with equality. Let $\phi(t) \geq x>\psi(t)$. Then we derive that $u(t, x) \geq$ $S_{\psi}(u)+S_{t^{\prime}}(u)$ as above, using now that $u(t, x)=g(t, x) \geq \frac{1}{2} g(t+\delta t, x+\sqrt{\delta t})+$ $\frac{1}{2} g(t+\delta t, x-\sqrt{\delta t})$.

Often the curves $\psi$ are the result of a shift in time or space applied to the curve $\phi$. We introduce the following notation. Let $\epsilon$ be a multiple of $\delta t$ and $\eta$ be a multiple of $\delta x$. Then we define $\phi_{\epsilon, \eta}$ by

$$
\phi_{\epsilon, \eta}(t)=\phi(t+\epsilon)+\eta .
$$

With respect to the function $g$ we define $g_{\epsilon, \eta}$ by

$$
g_{\epsilon, \eta}(t, x)=g(t+\epsilon, x+\eta) .
$$

\subsection{Differences in space and time}

We give now some estimates of differences in space and time of the solution of the free boundary problem (1) in the continuation region. They are stated in terms of corresponding differences in space and time of the prescribed function, which are known in principle. In fact we will compare expectations with respect to the free boundary, exactly equal to the solution, and expectations with respect to horizontal or vertical shifts of the free boundary, which are lower bounds of the solution. Below we suppose that $\phi$ is of minimal variation and weakly nondecreasing with $\phi(T)=0$. Let $(t, x) \in D$ with $x \geq \phi(t)$.

Differences in space. We start with upper and lower bounds for differences in space. Let $\eta \in \mathbb{X}, \eta>0$. Note that the curve $\phi_{0, \eta}$ lies in the continuation region and that for all $\tau$ with $t \leq \tau \leq T$

$$
P_{\phi_{0, \eta}}(t, x+\eta ; \tau, y+\eta)=P_{\phi}(t, x ; \tau, y) .
$$

Then it follows from (16) that

$$
u(t, x+\eta) \geq S_{\phi}\left(t, x ; g_{0, \eta}\right)+S_{T}\left(t, x ; g_{0, \eta}\right) .
$$

Since the curve $\phi_{0,-\eta}$ lies in the stopping region, it follows from (17) that

$$
u(t, x) \geq S_{\phi_{0,-\eta}}\left(t, x ; g_{0,-\eta}\right)+S_{T}\left(t, x ; g_{0,-\eta}\right) .
$$

Then (18) and (15) imply that

$$
u(t, x+\eta)-u(t, x) \geq S_{\phi}\left(t, x ; g_{0, \eta}-g\right)+S_{T}\left(t, x ; g_{0, \eta}-g\right)
$$

and (15) and (16) imply that

$$
u(t, x+\eta)-u(t, x) \leq S_{\phi_{0,-\eta}}\left(t, x ; g-g_{0,-\eta}\right)+S_{T}\left(t, x ; g-g_{0,-\eta}\right) .
$$


Differences in time. Let $\epsilon \in \mathbb{T}, \epsilon>0$. Let $t, x$ and $\epsilon>0$ be such that $(t, x),(t+$ $\epsilon, x) \in D$ and $x \geq \phi(t+\epsilon)$. Then by (15)

$$
u(t+\epsilon, x)=S_{\phi}(t+\epsilon, x ; g)+S_{T}(t+\epsilon, x ; g) .
$$

Also

$$
u(t, x)=S_{\phi}\left(t+\epsilon, x ; u_{-\epsilon, 0}\right)+S_{T}\left(t+\epsilon, x ; u_{-\epsilon, 0}\right) .
$$

This follows from (14), noting that the shift $\phi \mapsto \phi_{\epsilon, 0}$ leads to summation along a curve lying in the continuation region and that, when $\tau \geq \epsilon$ and $y \geq x$, the set of trajectories going from $(t+\epsilon, x)$ to $(\tau, y)$ is in bijection with the set of trajectories going from $(t, x)$ to $(\tau-\epsilon, y)$.

Applying (22) with $g=u$ and (23), we obtain the exact expression

$$
u(t+\epsilon, x)-u(t, x)=S_{\phi}\left(t+\epsilon, x ; u-u_{-\epsilon, 0}\right)+S_{T}\left(t+\epsilon, x ; u-u_{-\epsilon, 0}\right) .
$$

To obtain a lower bound for the difference in time, we note that by (23) and (16)

$$
u(t, x) \geq S_{\phi}\left(t+\epsilon, x ; g_{-\epsilon, 0}\right)+S_{T}\left(t+\epsilon, x ; g_{-\epsilon, 0}\right) .
$$

Hence by (22)

$$
u(t+\epsilon, x)-u(t, x) \leq S_{\phi}\left(t+\epsilon, x ; g-g_{-\epsilon, 0}\right)+S_{T}\left(t+\epsilon, x ; g-g_{-\epsilon, 0}\right) .
$$

To get a lower bound for the difference in time, we will obtain an upper solution for $u(t+\epsilon, x)$ by using Theorem 4 . Note that the curve $\phi_{-\epsilon, 0}$ lies in the stopping region and that for all $s$ with $t+\epsilon \leq s \leq T$

$$
P_{\phi_{-\epsilon}, 0}\left(t+\epsilon, x ; s, \phi(s-\epsilon)=P_{\phi}(t, x ; s, \phi(s)) .\right.
$$

Hence by Theorem 4

$$
u(t+\epsilon, x) \geq S_{\phi}\left(t, x ; g_{\epsilon, 0}\right)+S_{T-\epsilon}\left(t, x ; g_{\epsilon, 0}\right) .
$$

By (14)

$$
u(t, x)=S_{\phi}(t, x ; g)+S_{T-\epsilon}(t, x ; u)
$$

hence

$$
u(t+\epsilon, x)-u(t, x) \geq S_{\phi}\left(t, x ; g_{\epsilon, 0}-g\right)+S_{T-\epsilon}\left(t, x ; g_{\epsilon, 0}-u\right)
$$

\section{Continuous approximations of discrete formulas}

From now on we suppose that $\delta t \simeq 0$. Observe that also $\delta x=2 \sqrt{\delta t} \simeq 0$, but that $\delta x / \delta t=2 / \sqrt{\delta t}$ is infinitely large. We use $\oslash$ to designate an unknown or neglected infinitesimal, much like $o(1)$ designates an arbitrary function going to 0 . Limited numbers $(£)$ and appreciable numbers (@) are treated similarly. 


\subsection{Regularity of the binomial function}

We start by recalling a nonstandard extension of the DeMoivre-Laplace Theorem on the regularity properties of the function $b(t, x)$, which is useful in the study of the regularity properties of the solution of the free boundary property.

Theorem 5. (Higher order DeMoivre-Laplace Theorem, nonstandard [4]) For all $(t, x) \in$ $\Gamma$ such that $t$ is appreciable and $x$ is limited,

$$
b(t, x) \simeq \frac{1}{\sqrt{2 \pi t}} \exp \left(-\frac{x^{2}}{2 t}\right) \equiv G(t, x) .
$$

Moreover, for every standard $m, n \in \mathbb{N}$, one has for all $(t, x) \in \Gamma$ such that $t$ is appreciable and $x$ is limited

$$
b_{m, n}(t, x) \simeq \frac{\partial^{m} \partial^{n} G(t, x)}{\partial t^{m} \partial x^{n}} .
$$

Also is needed a bound on the binomial distribution, valid close to the origin and at the tails.

Theorem 6. For all $(t, x) \in \Gamma$ such that $t / \delta t$ is infinitely large

$$
b(t, x)=\frac{£}{\sqrt{t}} \exp \left(-@ \frac{x^{2}}{t}\right) .
$$

Proof. Assume first that $t / \delta t$ is even. If $x \geq 0$, by (7)

$$
\begin{aligned}
b(t, x) & =b(t, 0) \prod_{0 \leq y<x} \frac{b(y+\delta x)}{b(y)}=b(t, 0) \prod_{0 \leq y<x} \frac{1-\frac{y}{2 t} \delta x}{t+\frac{y}{2 t} \delta x+\frac{2}{t} \delta t} \\
& \leq b(t, 0) \prod_{0 \leq y<x} 1-\frac{y}{2 t} \delta x \leq b(t, 0) \exp -\sum_{0 \leq y<x} \frac{y}{2 t} \delta x \\
& \leq 2 b(t, 0) \exp -\int_{0}^{x} \frac{y}{2 t} \delta y \leq 2 b(t, 0) \exp -\frac{y^{2}}{4} .
\end{aligned}
$$

Also, one derives from the well-known asymptotical approximation $B\left(n, \frac{n}{2}\right) \sim \sqrt{\frac{2 \pi}{n}} 2^{n}$ for $n \rightarrow \infty$, that $b(t, 0)=\frac{(1+\oslash)}{\sqrt{2 \pi t}}$ for $t / \delta t$ infinitely large. Combining, we derive the bound (29). The case $x<0$ is obtained by symmetry. The case that $t / \delta t$ is odd follows from the above, noting that $b(t, x)=\frac{1}{2} b(t-\delta t, x+\sqrt{\delta t})+\frac{1}{2} b(t-\delta t, x-\sqrt{\delta t})$.

We need also bounds on the differences of the binomial function with respect to space. To this end we introduce an auxiliary function $c$, defined for $(t, x) \in D$ such that $x \geq 0$ by

$$
c(t, x)=\frac{x}{t+x \sqrt{\delta t}} .
$$


Note that by (8)

$$
b_{0,1}(t-\delta t, x-\sqrt{\delta t})=b(t-\delta t, x-\sqrt{\delta t}) c(t, x) .
$$

Lemma 7. Let $(t, x) \in D$ with $x \geq 0$, and $n \in \mathbb{N}, n \geq 1$ be standard. Then for all standard $j \in \mathbb{N}$

$$
c_{0, n}(t, x+j \delta x)=\frac{-\frac{n !}{t}\left(-\frac{\sqrt{\delta t}}{t}\right)^{n-1}}{\prod_{1 \leq i \leq n} 1+\frac{\sqrt{\delta t}}{t}(x+(j+n) \delta x)}
$$

Proof. Put $\gamma(z)=\frac{1}{1+z}$. Then it is shown by induction that its $n^{\text {th }}$ discrete derivative $\gamma_{n}$ satisfies

$$
\gamma_{n}(z)=\frac{(-1)^{n} n !}{\prod_{1 \leq i \leq n} 1+(z+n \delta x)}
$$

Observe that

$$
c(t, x+j \delta x)=\frac{1}{\sqrt{\delta t}}-\frac{1}{\sqrt{\delta t}} \frac{1}{1+\frac{\sqrt{\delta t}}{t}(x+j \delta x)} .
$$

Then the lemma follows by substitution.

Next lemma gives the bound in question.

Lemma 8. Let $(t, x) \in D$ with $x \gtrsim 0$, and $n \in \mathbb{N}, n \geq 1$ be standard. Then

$$
b_{0, n}(t-\delta t, x-\sqrt{\delta t})=£ \exp -@ \frac{x^{2}}{t} .
$$

Proof. For $t<x \sqrt{\delta t}$ we have $b(t-\delta t, x-\sqrt{\delta t})=0$. So let us consider some fixed $t \geq x \sqrt{\delta t}$, then $t / \delta t$ is unlimited. One proves by external induction from formula (30) that

$$
b_{0, n}(t-\delta t, x-\sqrt{\delta t})=P(t, x) b(t-\delta t, x-\sqrt{\delta t}) .
$$

where $P$ is a standard finite sum with limited coefficients of products of at most $n$ factors $c^{i}(t, x+j \delta x)$, with $1 \leq i, j \leq n$. It follows from Lemma 7 that these factors satisfy $c^{i}(t, x+j \delta x)=\frac{£}{t}$, so $P(t, x)=\frac{£}{t^{n}}$. Theorem 6 implies that $b(t-\delta t, x-\sqrt{\delta t})=$ $£ \exp -@ \frac{x^{2}}{t}$. Hence

$$
b_{0, n}(t-\delta t, x-\sqrt{\delta t})=\frac{£}{t^{n}} £ \exp -@ \frac{x^{2}}{t}=£ \exp -@ \frac{x^{2}}{t}
$$




\subsection{Estimates of probabilities of transition}

We turn now to infinitesimal approximations of path sums. We consider the probability of transition to the curve $\lambda: \mathbb{T} \rightarrow R$ defined by $\lambda(t)=\sqrt{\delta t}-(-1)^{t / \sqrt{\delta t}} \sqrt{\delta t}$.

Proposition 9. Let $(0, x) \in D$ with $x \gtrsim 0$ and $t \in \mathbb{T}$ be such that $t>0, t \simeq 0$. Then the probability of a first hit of $\lambda$ before $t$ is infinitesimal.

Proof. The first possibility of hitting $\lambda$ is at time $x \sqrt{\delta t}$, which is infinitely large with respect to $\delta t$. Applying Proposition 3 and Theorem 6 we find, for some standard $K$ and $C$

$$
\begin{aligned}
\sum_{0 \leq \tau \leq t} P(0, x ; t, 0) & =\sum_{x \sqrt{\delta t} \leq \tau \leq t} b(\tau-\delta t, x-\sqrt{\delta t}) \frac{x}{\tau+x \sqrt{\delta t}} 2 \delta t \\
& \leq \int_{x \sqrt{\delta t}}^{t} \frac{K}{\sqrt{s}} \exp \left(-C \frac{x^{2}}{s}\right) \frac{x}{s+x \sqrt{\delta t}} d s \\
& \leq K \int_{0}^{t} \exp \left(-C \frac{x^{2}}{s}\right) \frac{x}{s \sqrt{s}} d s \\
& \leq K \frac{x}{\sqrt{t}} \exp \left(-C \frac{x^{2}}{t}\right) \\
& \simeq 0 .
\end{aligned}
$$

Proposition 10. Let $(0, x) \in D$ with $x \simeq 0$. Then the probability of not hitting $\lambda$ before an appreciable time $t$ is infinitesimal, in fact it satisfies

$$
\sum_{y>0} P(0, x ; t, y)=(1+\oslash) x \sqrt{\frac{2}{\pi t}}
$$

Proof. Because the function $b$ is at least of class $S^{1}$, it follows from the Higher order DeMoivre-Laplace Theorem that for limited $y$

$$
\frac{b(t, y-x)-b(t, y+x)}{2 x} \simeq-\frac{\partial\left(\frac{1}{\sqrt{2 \pi t}} \exp \left(-\frac{y^{2}}{2 t}\right)\right)}{\partial y}=\frac{y}{\sqrt{2 \pi} t \sqrt{t}} \exp \left(-\frac{y^{2}}{2 t}\right) .
$$


It follows from Lemma 1 that for positive unlimited $y$

$$
\begin{aligned}
\frac{b(t, y-x)-b(t, y+x)}{2 x} & =-\frac{1}{2 x} \sum_{-x \leq z<x} b_{0,2}(t, y-z) \delta x \\
& \leq-\frac{1}{2 x} \sum_{-x \leq z<x} b_{0,2}(t, y-x) \delta x \\
& =-b_{0,2}(t, y-x) \\
& =(1+\oslash) b(t, y-x) \frac{y}{t+y \sqrt{\delta t}}
\end{aligned}
$$

It follows from Theorem 6 that the latter expression is less than $K \exp \left(-C y^{2}\right)$ for some standard $K, C>0$. Hence we may apply the Lemma of Dominated Approximation [1, p. 142] to derive that

$$
\begin{aligned}
\sum_{y>0} P(0, x ; t, y) & =\sum_{y>0}(b(t, y-x)-b(t, y+x)) \delta x \\
& =2 x \sum_{y>0} \frac{b(t, y-x)-b(t, y+x)}{2 x} \delta x \\
& =(1+\oslash) 2 x \sum_{y>0} \frac{y}{\sqrt{2 \pi} t \sqrt{t}} \exp \left(-\frac{y^{2}}{2 t}\right) \delta x \\
& =(1+\oslash) \frac{2 x}{\sqrt{2 \pi t}} \int_{0}^{\infty} \frac{y}{\sqrt{t}} \exp \left(-\frac{y^{2}}{2 t}\right) d\left(\frac{y}{\sqrt{t}}\right) \\
& =(1+\oslash) x \sqrt{\frac{2}{\pi t}},
\end{aligned}
$$

which is infinitesimal.

Proposition 11. Let $(0, x) \in D$ with $x \simeq 0$. Then there exists $t \in \mathbb{T}, t \simeq 0$ such that the probability of the first hit of $\lambda$ before time $t$ is nearly equal to 1.

Proof. By Proposition 10 the probability of not hitting $\lambda$ before some appreciable time is infinitesimal. By the Fehrele Principle this remains true for some $t \simeq 0$. Then the probability of the first hit of $\lambda$ before $t$ is nearly equal to 1 .

The above property remains true if we consider the first hit of a weakly nondecreasing curve of minimal variation $\phi$ instead of $\lambda$, because a trajectory hitting $\lambda$ will hit $\phi$ before. This is stated in the next proposition. 
Proposition 12. Let $(0, x) \in D$ with $x \simeq 0$. Let $\phi: \mathbb{T} \rightarrow \mathbb{R}$ be a weakly nondecreasing function of minimal variation such that $\phi(0)=0$. Then there exists $t \in \mathbb{T}, t \simeq 0$ such that the probability of the first hit of $\phi$ before time $t$ is nearly equal to 1.

\subsection{Estimates of path sums}

We present some lemmas on the infinitesimal approximation of path sums with respect to some function $f$, along a curve $\phi$ and/or along the horizon. They assume some regularity of $f$ in the limited domain, and some growth condition at positive infinity. In the next sections they will be applied to obtain estimates of solutions, or differences of solutions. We start with some estimates along the horizon.

Lemma 13. Let $\omega \simeq+\infty$. Let $f$ be a real function such that $|f(y)| \leq K \exp (C y)$ for $y \geq A$, where $A, C$ and $K$ are standard constants. Let $\phi:[0 \cdot T] \rightarrow \mathbb{R}$ be a $S$-continuous and weakly nondecreasing function of minimal variation with $\phi(T)$ limited. Then for $0 \leq t<T$ and limited $x>\phi(t)$

$$
\sum_{y>\omega} P(t, x ; T, y) f(y) \simeq 0 .
$$

Also $S_{T}(t, x ; f)$ is limited. Moreover, if the probability of hitting the horizon is infinitesimal, it holds that $S_{T}(t, x ; f) \simeq 0$.

Proof. We have $P(t, x ; T, y) \leq b(T-t, y-x) \delta x$. If $(T-t) / \delta t$ is limited, there are no trajectories hitting the horizon above $\omega$, so (31) holds trivially. If $(T-t) / \delta t$ is unlimited, by applying Theorem 6 we see that $b(T-t, \omega-x)$ is infinitesimal, since it is of the form $\frac{£}{\sqrt{t}} \exp \left(-@ \frac{(\omega-x)^{2}}{t}\right)$, while

$$
b(T-t, y-x)|f(y)| \leq \frac{£}{\sqrt{t}} \exp \left(-@ \frac{(y-x)^{2}}{t}\right) K \exp (C y)
$$

is of exponential decay. Then (31) is a consequence.

Let $a>0$ be appreciable. Applying the Cauchy principle to (31), we see that $\sum_{y>\omega} P(t, x ; T, y)|f(y)| \leq a$ for some limited $\omega_{0}$. Then, noting that $f$ is limited on $\left[\phi(T) \cdots \omega_{0}\right]$, we obtain that $S_{T}(t, x ; f)$ is limited. Finally, if the probability of hitting the horizon is infinitesimal one has $\sum_{\phi(T)<y \leq z} P(t, x ; T, y) f(y) \simeq 0$ for all limited $z$, so by Robinson's Lemma $\sum_{\phi(T)<y \leq \omega} P(t, x ; \bar{T}, y) f(y) \simeq 0$ for some $\omega \simeq+\infty$. Then (31) implies that $S_{T}(t, x ; f) \simeq 0$.

The first part of next lemma states that, starting infinitely close to a curve, a path sum with respect to a function along the curve is nearly equal to the initial value on the curve, if the function is $S$-continuous. The second part states that, if this initial value is 
infinitesimal, and the function remains infinitesimal, except possibly on the unlimited part of horizon, where it should satisfy some growth condition, the path sum is also not more than infinitesimal.

Lemma 14. Let $\omega \simeq+\infty$. Let $\phi:[0 \cdot T] \rightarrow \mathbb{R}$ be a weakly nondecreasing function of minimal variation of class $S^{0}$. Let $t \lesssim T$ and $f:[t, T] \times \mathbb{R} \rightarrow \mathbb{R}$.

1) If $f$ is of class $S^{0}$ and such that there exist standard constants $A, C$ and $K$ such that $|f(T, y)| \leq K \exp (C y)$ for $y \geq A$, for $x \simeq \phi(t), x>\phi(t)$

$$
S_{\phi}(t, x ; f)+S_{T}(t, x ; f) \simeq f(t, \phi(t)) .
$$

2) Assume $f(\tau, \phi(\tau) \simeq 0$ for all $\tau \in[t, T]$ and $f(T, y) \simeq 0$ for all limited $y \geq$ $\phi(T)$, and there exist standard constants $A, C$ and $K$ such that $|f(T, y)| \leq K \exp (C y)$ for all $y \geq A$. Then for limited $x>\phi(t)$

$$
S_{\phi}(t, x ; f)+S_{T}(t, x ; f) \simeq 0 .
$$

Proof. 1) By Proposition 11 there exists $t^{\prime} \simeq t, t^{\prime} \geq t$ such that that the probability to hit $\phi$ at some $\tau$ with $t \leq \tau \leq t^{\prime}$ is nearly equal to 1 . Since $f(\tau, \phi(\tau)) \simeq f(t, \phi(t))$ for such $\tau$, and $f(\tau, \phi(\tau))$ remains limited for $t^{\prime} \leq \tau \leq T$,

$$
\begin{aligned}
S_{\phi}(t, x ; f) & \simeq \sum_{t \leq \tau \leq t^{\prime}} P_{\phi}(t, x ; \tau, \phi(\tau)) f(\tau, \phi(\tau)) \\
& \simeq \sum_{t \leq \tau \leq t^{\prime}} P_{\phi}(t, x ; \tau, \phi(\tau)) f(t, \phi(t)) \simeq f(t, \phi(t)) .
\end{aligned}
$$

Then the result follows from Lemma 13 .

2) We have $S_{\phi}(t, x ; f) \simeq 0$ because always $f(\tau, \phi(t)) \simeq 0$. Then the result follows from Lemma 13.

The first part of the last lemma states that, at limited points infinitely close to the horizon, the value of the path sum with respect to a $S$-continuous function $f$ satisfying a growth condition is infinitely close to the value of $f$ at the horizon. The second and third part state that these path-sums globally respect the growth conditions on $f$.

Lemma 15. Let $\phi:[0 \cdot T] \rightarrow \mathbb{R}$ be a weakly nondecreasing function of minimal variation of class $S^{0}$. Let $f:[0, T] \times \mathbb{R}$ be a $S$-continuous real function such that there exist standard constants $A, C$ and $K$ such that $|f(T, y)| \leq K \exp (C y)$ for $y \geq A$. Then

1) $S_{\phi}(t, x ; f)+S_{T}(t, x ; f) \simeq f(T, x)$ for $t \simeq T$ and $x>\phi(t)$ limited.

2) $S_{\phi}(t, x ; f)+S_{T}(t, x ; f)$ is limited for all $t$, and limited $x>\phi(t)$.

3) $S_{\phi}(t, x ; f)+S_{T}(t, x ; f) \leq 2 K \exp (2 C x)$ for all $t$, and for all $x>\phi(t)$. 
Proof. 1) First assume that $x \gtrsim \phi(T)$ is limited. If $(T-t) / \delta t$ is standard, all trajectories $\lambda$ of $\Lambda_{t, x}$ satisfy $\lambda(T) \simeq x$. Then $f(T, \lambda(T)) \simeq f(T, x)$ and taking the mean over these values we clearly have $S_{\phi}(t, x ; f)+S_{T}(t, x ; f)=S_{T}(t, x ; f) \simeq$ $f(T, x)$. If $t \simeq T$ with $(T-t) / \delta t$ unlimited, by the estimate of Theorem 6 , for some all appreciable $\alpha>0$ the probability that $|\lambda(T)-x| \leq \alpha$ is infinitesimal. By the Fehrele principle this is still true for some $\alpha \simeq 0$. Since we still have $\eta(T, \lambda(T)) \simeq f(T, x)$ for these $\lambda, \sum_{x-\alpha \leq y \leq x+\alpha} P(t, x ; T, y) f(y) \simeq f(T, x)$. By Lemma $13 \sum_{x+\alpha \leq y} P(t, x ; T, y) f(y) \simeq 0$. Because $f$ is limited for limited arguments one has both $\sum_{\phi(T)<y<x-\alpha} P(t, x ; T, y) f(y) \simeq 0$ and $S_{\phi}(t, x ; f) \simeq 0$. Combining, we derive that $S_{\phi}(t, x ; f)+S_{T}(t, x ; f) \simeq f(T, x)$. The case where $x \simeq \phi(T), x \geq \phi(t)$ is similar to the case $x \gtrsim \phi(T)$ : there exists $\alpha^{\prime}>0, \beta^{\prime} \simeq 0$ such that for trajectories starting at $(t, x)$ the probability that $\lambda(T)-x \geq \alpha^{\prime}$ is less then $\beta^{\prime}$. So with probability nearly equal to 1 the remaining trajectories hit the horizon or the free boundary at points where $u$ takes values infinitely close to $f(T, \phi(T)) \simeq f(T, x)$. Hence $S_{\phi}(t, x ; f)+S_{T}(t, x ; f) \simeq f(T, x)$.

2) Since $f(\tau, \phi(t))$ is limited for all $\tau$ with $t \leq \tau \leq T$, for $x>\phi(t)$ clearly $S_{\phi}(t, x ; f)$ is limited. By Lemma 13 also $S_{\phi}(t, x ; f)$ is limited. Hence $S_{\phi}(t, x ; f)+$ $S_{T}(t, x ; f)$ is limited.

3) If $x$ is unlimited, the values $|f(T, \lambda(T))|$ such that $0 \leq \lambda(T) \leq 2 x$ for $\lambda \in \Lambda_{t, x}$ are bounded by $K \exp (2 C x)$. Using Theorem 6 we find that for some standard $A$ and $B$,

$$
\begin{aligned}
& \sum_{z \geq 2 x} P(T-t, x ; T, x+z)|f(T, x+z)| \delta x \\
\leq & \sum_{z \geq 2 x} b(T-t, z)|f(T, x+z)| \delta x \\
\leq & A \int_{z \geq 2 x} \exp \left(-B z^{2}\right) K \exp (2 C(x+z)) d z \simeq 0 .
\end{aligned}
$$

Hence $S_{\phi}(t, x ; f)+S_{T}(t, x ; f) \leq 2 K \exp (2 C x)$.

\section{Hypotheses, some examples, earlier approaches}

From now on we assume the following hypotheses.

Hypotheses 16. 1) The horizon $T>0$ is standard and $\delta t \simeq 0$.

2) The fixed condition $g:[0, T] \times \mathbb{R} \rightarrow \mathbb{R}$ is standard and of class $C^{1,2}$, except maybe at the non-negative part of the horizon. At the horizon the function $g(T,.) \equiv h$ is 
nonnegative, with $g(T, 0)=0$. At $(T, 0)$, it may be not two times differentiable in space, and when $x$ is positive, it is two times continuously differentiable with respect to $x$; the function and its partial derivatives do not need to be continuous in $t$ if $(t, x)$ approaches $(T, x)$, but their limits are supposed to exist. Also $|g|,\left|\frac{\partial g}{\partial t}\right|,\left|\frac{\partial g}{\partial x}\right|$ and $\left|\frac{\partial^{2} g}{\partial x^{2}}\right|$ have at most standard exponential growth in $x$ at $+\infty$, for all $t$ such that $0 \leq t \leq T$.

3) The function $g$ satisfies $\frac{\partial g(t, x)}{\partial t}<-\frac{1}{2} \frac{\partial^{2} g(t, x)}{\partial x^{2}}$, except possibly at the nonnegative part of the horizon.

4) The continuation region $C$ is on top of the stopping region $S$, their boundary $\phi$ is supposed to be included in $S$, terminating at the horizon at $(T, 0)$, and to be a weakly nondecreasing function of time.

Condition 16.2 implies that the restriction of $g$ to $D$ is of class $S^{1,2}$, with possible exceptions infinitely close to the positive part of the horizon; these express that the condition at the horizon is in principle to be seen apart from the overall behavior of the prescribed function $g$. Also the discretisations $g, g_{1,0}, g_{0,1}$ and $g_{0,2}$ have standard exponential growth at infinity.

As regards to Condition 16.3, since the solution satisfies the martingale property in the continuation region, it is natural to suppose that the prescribed function does not satisfy the martingale property in the stopping region. If so, (3) implies that

$$
g(t, x)>\frac{1}{2} g(t+\delta t, x+\sqrt{\delta t})+\frac{1}{2} g(t+\delta t, x-\sqrt{\delta t}) .
$$

Next proposition states that (32) follows from the inequality of Condition 16.3. In fact we have two stronger inequalities.

Proposition 17. Let $(t, x) \in S$, with $x$ limited. Then

$$
g_{1,0}(t, x) \succsim-\frac{1}{2} g_{0,2}(t, x),
$$

provided $t \leq T-2 \delta$ t and $x<-\delta x$, as long as $t \simeq T$. Moreover for $0<t<T$ and $x<0$ limited one has (32), in fact

$$
g(t, x)-\left(\frac{1}{2} g(t+\delta t, x+\sqrt{\delta t})+\frac{1}{2} g(t+\delta t, x-\sqrt{\delta t})\right)=@ \delta t .
$$

Proof. Noting that $\phi$ is of minimal variation, also $(t, x-2 \delta x),(t, x-\delta x),(t-\delta t, x-$ $3 \sqrt{\delta t}),(t-\delta t, x-\sqrt{\delta t})$ and $(t-2 \delta t, x-\delta x)$ lay in $S$. Then

$$
\frac{1}{4} g(t, x-2 \delta x)+\frac{1}{2} g(t, x-\delta x)+\frac{1}{4} g(t, x)<g(t-2 \delta t, x-\delta x),
$$

implying that $g_{0,1}(t-2 \delta t, x-\delta x)<-\frac{1}{2} g_{0,2}(t-2 \delta t, x-2 \delta x)$. If we had near-equality, it would follow that $\frac{1}{2} g_{2}^{\prime \prime}\left({ }^{\circ} t,{ }^{\circ} x\right)=-g_{1}^{\prime}\left({ }^{\circ} t,{ }^{\circ} x\right)$, a contradiction. Hence $g_{0,1}(t-2 \delta t, x-$ 
$\delta x) \succsim-\frac{1}{2} g_{0,2}(t-2 \delta t, x-2 \delta x)$. By $S$-continuity of the discrete derivatives, also $g_{0,1}(t, x) \underset{ }{\gtrsim}-\frac{1}{2} g_{0,2}(t, x)$, with $g_{0,1}(t, x)$ well-defined for $t+2 \delta t \leq T$; also the definition of $g_{0,2}(t, x)$ uses only points of non-negative $x$-values if $x \leq-\delta x$.

As for the second part, using a Taylor expansion, we find

$$
\begin{aligned}
& \frac{g(t+\delta t, x+\sqrt{\delta t})+g(t+\delta t, x-\sqrt{\delta t})}{2} \\
= & g(t, x)+\left(\frac{\partial g(t, x)}{\partial t}+\frac{1}{2} \frac{\partial^{2} g(t, x)}{\partial x^{2}}+\oslash\right) \delta t=g(t, x)-@ \delta t .
\end{aligned}
$$

This proves the Proposition.

As regards to Condition 16.4, it states firstly that the continuation region and the stopping region are separated by a discrete curve. Usually it is assumed that the continuation region and the stopping region are separated by some curves, so in a sense we are studying the free boundary problem locally. Then it is no essential restriction to suppose that the stopping region lies below the continuation region.

As pointed out in Subsection 3.2, being weakly nondecreasing is a fairly natural condition to impose on discretisations of nondecreasing functions. The condition that the free boundary is weakly nondecreasing is an essential restriction, but will be satisfied if the condition at the horizon is sufficiently rapidly increasing for positive arguments, such as happens for instance in the examples below. In fact it is only needed infinitely close to the boundary. Then by permanence the free boundary will be weakly nondecreasing during a time interval $\left[T-T_{0} . . T\right]$ of appreciable length. We may consider the free boundary problem only locally, and then it is possible to suppose that the free boundary $\phi$ is weakly nondecreasing during the whole time interval of definition. In fact it is only needed to ask that the free boundary is weakly nondecreasing as a curve.

Lemma 18. The free boundary $\phi$ is functional, in fact of minimal variation as long as $(t, \phi(t)$ is limited.

Proof. It follows from (32) that whenever $x<0$ is limited and $t>0$, if $u(t+\delta t, x+$ $\sqrt{\delta t})=g(t+\delta t, x+\sqrt{\delta t})$, then also $u(t, x)=g(t, x)$. Hence in case $(t+\delta t, x+\sqrt{\delta t}) \in \phi$, since $\phi$ is weakly nondecreasing, we can only have $(t+\delta t, x) \in \phi$ or $(t+\delta t, x+2 \sqrt{\delta t}) \in$ $\phi$, and one cannot have both. Hence $\phi$ is functional. This implies that it is of minimal variation: if not, for some $t$ the points $(t, \phi(t))$ and $(t, \phi(t)-\delta x)$ lie on the boundary of $S$, so the free boundary cannot be functional.

Here we present some simple examples.

Example 1. Define $h(x)=x$ for $x \geq 0$ and $g(t, x)=0$, except for $t=T$ and $x \geq 0$. Then $\phi(t)=-(T-t) / \sqrt{\delta t}$ for $t \geq 0$, for always $u(t, \phi(t)+\delta x)=\mu(t, \phi(t)+\delta x)=$ $\delta x / 2^{(T-t) / \delta t}$, while $u(t, \phi(t))=g(t, \phi(t))=0$. The function $\phi$ is not $S$-continuous, in fact its shadow is the non-positive part of the line $x=T$. 
Example 2. Define $h(x)=-x$ for $x \leq 0$ and $g(t, x)=0$, except for $t=T$ and $x \leq 0$. Here the stopping region lies on top of the continuation region, with free boundary $\phi(t)=(T-t) / \sqrt{\delta t}$ for $t \geq 0$. In fact the problem is symmetric with respect to the first example.

Example 3. Define $h(x)=x^{2}$ for $x \geq 0$ and $g(t, x)=T-t$, except for $t=T$ and $x \leq 0$. Then $\phi(t)=\sqrt{\delta t}-(-1)^{T / \delta t} \sqrt{\delta t}$, so the free boundary is $S$-continuous, with shadow identically zero. In fact one proves by backward induction that $u(t, x)=$ $\mu(t, x)=x^{2}+T-t$ for $x>0$, while for $x=0$

$$
\mu(t, 0)=\frac{1}{2}\left(\delta t+(T-t-\delta t)+\frac{1}{2}(T-t-\delta t)=T-t-\frac{1}{2} \delta t .\right.
$$

As a consequence $u(t, x)=g(t, x)=T-t$ for $x \leq 0$.

Example 4. Define $g(t, x)=x^{3}$ for $x \geq 0$, and $g(t, x)=3(T-t) x$ for $x<0$. Again we have a $S$-continuous free boundary defined by $\phi(t)=\sqrt{\delta t}-(-1)^{T / \delta t} \sqrt{\delta t}$. One proves by backward induction that $u(t, x)=\mu(t, x)=x^{3}+3(T-t) x$ for $x>0$, while $u(t, x)=g(t, x)=0$ for $x=0$, because

$$
\begin{aligned}
\mu(t, 0) & =\frac{1}{2}\left(\delta t^{3 / 2}+3(T-t-\delta t) \delta t^{1 / 2}+\frac{1}{2}\left(-\delta t^{3 / 2}+3(T-t-\delta t)\left(-\delta t^{1 / 2}\right)\right.\right. \\
& =g(t, 0)=0
\end{aligned}
$$

Then also $u(t, x)=g(t, x)=T-t$ for $x \leq 0$, hence $\phi$ divides the continuation region and stopping region indeed.

The first two examples suggest that if the condition at the horizon is continuous, but not differentiable at 0 , the free boundary tends to be at least locally almost parallel to the horizon. In Example 3 the free boundary is perpendicular to the horizon if the the prescribed function at the horizon is once, but not two times differentiable at 0 , otherwise it is smooth and satisfies locally the backward heat equation; observe that $\frac{1}{2} \frac{\partial x^{2}}{\partial x}=1=$ $-\frac{\partial(T-t)}{\partial t}$.

We may have a free boundary $\phi$ between these extremes if the prescribed function locally satisfies "almost" the backward heat equation, meaning that some terms of higher order are added to $x^{2}$ respectively $T-t$. Observe that due to the fluctuations of order $\sqrt{\delta t}$ at time-scale $\delta t$, the function $\phi$ may only be $S$-continuous, and never can be $S$-differentiable, though ${ }^{0} \phi$ may be differentiable. This is achieved when $\phi$ is $S$-differentiable at the mesotimescale $\sqrt{\delta t}$. To illustrate what is bound to happen if $0<{ }^{0} \phi^{\prime}(T)<\infty$, to fix ideas we consider the case where ${ }^{0} \phi^{\prime}(T)=1$. In the most simple situation we have the following phenomena at micro-scale $\delta t$ and meso-scale $\sqrt{\delta t}$.

Starting at $T$ with a sufficiently regular final condition $h$, working backwards $\phi(t)$ takes alternately the values 0 and $-\sqrt{\delta t}$, up to some time $T-\tau_{1}$ with $\tau_{1} / \sqrt{\delta t} \simeq 1$. 
At $T-\tau_{1}$ and $T-\tau_{1}-\delta t$ it takes (backwards) two downward steps $-\sqrt{\delta t}$. We now have $\left(\phi(T)-\phi\left(T-\tau_{1}\right)\right) / \tau_{1} \simeq 1$. We find a second time $\tau_{2}$ such that $\left(\phi\left(T-\tau_{1}\right)-\right.$ $\left.\phi\left(T-\tau_{2}\right)\right) /\left(\tau_{2}-\tau_{1}\right) \simeq 1$, if $\phi(t)$ takes alternately the values $-\sqrt{\delta t}$ and $-2 \sqrt{\delta t}$, up to some time $T-\tau_{2}$ with $\tau_{2} / \sqrt{\delta t} \simeq 1$, when it takes again two downward backward steps $-\sqrt{\delta t}$. This procedure should be repeated as long as the times $\tau$ remain infinitesimal.

During this process we should be able to calculate or approximate with small error the solution $u$ close to $\phi$. Though calculations rapidly may grow in complexity, we note that, within the "levels" of alternate behavior, i.e. from $T$ to $T-\tau_{1}$, and $T-\tau_{1}$ to $T-\tau_{2}$, etc. one could take profit of the reflection principle, and even of asymptotic estimates as in Section 5 , since the number of micro-timesteps $\delta t$ on these levels is infinitely large; at time $T-\tau_{1}$ one could start all over again with final condition $h_{1} \equiv u\left(T-\tau_{1}, x\right)$, in the hope that $h_{1}$ has a Taylor expansion similar to $h$, after making a change of variables $\left(\tau_{1}, 0\right) \mapsto$ $(0,-\sqrt{\delta t})$. The author was able to carry out this process in one special case $(h(x)=$ $x^{2}+\frac{2}{3} x^{3}$ ), already with rather complex computations. For this repeated procedure to have success, investigation and error-analysis is needed which lies outside the scope of this article.

Important contributions to the study of the regularity of the free boundary and the solution have been made by Van Moerbeke [11][12] and Friedman [7]. We will briefly compare their results to our approach, guided by the special case where $g(t, x)=T-t$, as in [11], and the free boundary in the form of one curve $\phi$. As in example 3 we will suppose that the condition at the horizon $h$ satisfies $h(x)=0$ for $x \leq 0$, otherwise is three-times differentiable, with $h^{\prime}(0)=0$ and $h^{\prime \prime}(0)=2$ (Van Moerbeke and Friedman consider the case of a finite number of such curves, starting at well-isolated points satisfying these two equalities). This means that the Taylor-expansion of $h$ starts with the quadratic term $x^{2}$, with possibly higher-order terms. Observe that at $(T, 0)$, the couple $(g, h)$ satisfies the backward heat equation, for $\frac{\partial g}{\partial t}+\frac{1}{2} \frac{d^{2} h}{d x^{2}}=0$. Using continuous-time stochastics [11] shows that, starting at $(T, 0)$, the free boundary is differentiable, down to a time $t_{0}$, where the derivative possibly goes to infinity; this time is related to points at the horizon where $h^{\prime \prime}$ and $h^{\prime \prime}-2$ changes sign, otherwise said the condition at the horizon switches from convexity to concavity respectively from locally minorized by the quadratic function, or majorized by it. The proof uses a result on local differentiability of [12, published later] established under more general conditions. The function $g$ should have partial derivatives up to order 4 , and, together with the partial derivatives up to order 3 , should satisfy so-called Tychonov growth conditions when $x$ goes to infinity, meaning that they should be at most of order $\exp \left(o\left(x^{2}\right)\right)$. Then the free boundary is differentiable at least in a neighborhood of $(T, 0)$. The rather involved proof uses an integral equation for $\phi$. Once this is established, regularity properties of the solution $u$ are shown which are very similar to the properties shown in the remaining sections: $u$ is $C^{\infty}$ at points away from the boundary and the horizon, at the boundary it is $C^{1}$, but not $C^{2}$ etc. Like in [12] differentiability of $\phi$ is not really needed, essential is that $\phi$ is continuous, comparable to our result of $S$-continuity (see Subsection 7.1). This result is not as strong as ( $S$ - 
)differentiability, but we note that we imposed less smoothness on the function $g$; the choice of the conditions of linear exponential growth, instead of sub-quadratic exponential growth, is more a matter of convenience and it is not difficult adapt to our proofs to this more general growth condition.

The article [7] is written in a different, but largely equivalent context, using parabolic partial differential equations and distributions. It presents some alternative proofs and refinements of Van Moerbeke's results, while in most cases it the only smoothness condition on $g$ and $h$ needed is that they are $C^{2}$. The free boundary is locally monotone, and in the above special case, it starts being decreasing when going backwards in time, as is being assumed in the present article. The regularity properties of the solution are first established in this context, then also for the increasing case. Friedman also establishes a differential equation for $\phi$, which is implicit and uses simultaneously a second-order partial derivative of the solution. As in Van Moebeke's article the arguments are rather involved, improving gradually the regularity properties obtained.

Summarizing, our results are not so good as those by Van Moerbeke and Friedman, yet contain a substantial part of it, and were obtained in a finite setting by straightforward, still rather comprehensive, calculations.

\section{7. $S$-continuity of the free boundary and the solution}

In this section we prove first that the free boundary is $S$-continuous at least during a time-interval of appreciable length. Then without restriction of generality the free boundary may be supposed to be $S$-continuous throughout. We prove also that the solution $u$ is of class $S^{0}$ in the continuation domain. Now by Lemma 15 the path sum $u$ is limited in the limited domain, so we focus on $S$-continuity. The proof uses some "domain decomposition": we distinguish cases where we are infinitely close to the horizon, infinitely close to the free boundary, or at appreciable distance to them. In the next section it is shown that $u$ has much more regularity than only $S$-continuity, but the proof will need that $S$-continuity has already been established.

\section{1. $S$-continuity of the free boundary}

We will establish the $S$-continuity property progressively. Lemma 22 says that the free boundary is $S$-continuous infinitely close to the horizon. Theorem 19 extends the $S$-continuity of the free boundary to at least an appreciable interval. The proof of Lemma 22 includes some careful estimates, partly carried out in the proofs of Lemma 20 and Lemma 21. We resume the results as follows.

Theorem 19. The free boundary $\phi$ is of minimal variation and $S$-continuous from the horizon at least during a time-interval of appreciable length.

In order to prove that the free boundary is $S$-continuous at the horizon, we give now some preparatory results on behavior close to the negative part the horizon. As is ex- 
pected, the influence of the condition $h$ at the positive part of the horizon is absent or very reduced.

Lemma 20. Assume $x<0$ is limited. Then $u(t, x)=g(t, x)$ for $t \geq T+x \sqrt{\delta t}$.

Proof. If $x \leq-(T-t) / \sqrt{\delta t}$, the function $h$ cannot influence $u(t, x)$, for no trajectory of the Wiener walk starting at $(t, x)$ can hit the horizon at a point of the form $(T, y)$ with $y>0$. For $(\tau, \xi)$ such $|\xi-x| \leq(\tau-t) / \sqrt{\delta t}$ we have $\frac{1}{2} g(\tau+\delta t, \xi-\sqrt{\delta t})+\frac{1}{2} g(\tau+$ $\delta t, \xi+\sqrt{\delta t})<g(\tau, \xi)$. Hence $u(t, x)=g(t, x)$.

Lemma 21. Assume $x \gtrsim 0$ is limited and $t \simeq T, T-t>-x \sqrt{\delta t}$. Let $\phi:[t \cdot T]$ be weakly nondecreasing such that $\phi(t)=x-\sqrt{\delta t}$ and $\phi(T)=0$. Then both

$$
S_{T}(t-\delta t, x ; h)=\oslash \delta t
$$

and

$$
S_{T-\delta t}(t-\delta t, x+\sqrt{\delta t} ; g)=\oslash \delta t
$$

Proof. By Theorem 6

$$
\begin{aligned}
P(t-\delta t, x ; T, y) & \leq b(T-t-\delta t, y-x) \delta x \\
& =\frac{£}{\sqrt{T-t-\delta t}} \exp \left(-@ \frac{(y-x)^{2}}{T-t-\delta t}\right) \delta x .
\end{aligned}
$$

Since $h(y) \leq K e^{C y}$ for all $y>0$, where $K$ and $C$ are standard positive constants, and $(y-x)^{2}$ is at least appreciable for $y>0$, we derive an estimation of the form

$$
\sum_{y>0} P(t, x ; T, y) h(y)=\frac{£}{\delta t^{1 / 4}} \exp \left(-\frac{@}{\sqrt{\delta t}}\right) .
$$

This is clearly infinitely small with respect to $\delta t$. Noting that $g(T-\delta t, y)$ satisfies the same growth condition as $h$, the second estimation is proved similarly.

Lemma 22. Let $t \simeq T$. Then $\phi(t) \simeq 0$.

Proof. Let $x \gtrsim 0$ be limited. Note that $u(T, x)=g(T, x)$. Let $t$ be minimal such that still $u(t, x)=g(t, x)$. Then $T-t \geq-x / \sqrt{\delta t}$ by Lemma 20. Suppose $t \simeq T$. Then $u(t-2 \delta t, x)=\mu(t-2 \delta t, x)$, and it follows from (32) that $u(t-\delta t, x-\sqrt{\delta t})=$ $g(t-\delta t, x-\sqrt{\delta t})$ and $u(t-\delta t, x+\sqrt{\delta t})=\mu(t-\delta t, x+\sqrt{\delta t})$. Now

$$
g(t-\delta t, x+\sqrt{\delta t}) \geq \mu(t-\delta t, x+\sqrt{\delta t})+\oslash \delta t,
$$

Indeed, by Lemma 21

$$
\begin{aligned}
\mu(t-\delta t, x+\sqrt{\delta t}) & =S_{\phi}(t-\delta t, x+\sqrt{\delta t} ; g)+S_{T}(t-\delta t, x+\sqrt{\delta t} ; h) \\
& =S_{\phi}(t-\delta t, x+\sqrt{\delta t} ; g)+\oslash \delta t .
\end{aligned}
$$


Also, because $g$ satisfies (3) up to $T-\delta t$, again by Lemma 21

$$
\begin{aligned}
& g(t-\delta t, x+\sqrt{\delta t}) \geq S_{\phi}(t-\delta t, x+\sqrt{\delta t} ; g)+S_{T-\delta t}(t-\delta t, x+\sqrt{\delta t} ; g) \\
= & S_{\phi}(t-\delta t, x+\sqrt{\delta t} ; g)+\oslash \delta t .
\end{aligned}
$$

Then it follows from Proposition 17 that

$$
\begin{aligned}
g(t-2 \delta t, x) & =\frac{1}{2} g(t-\delta t, x+\sqrt{\delta t})+\frac{1}{2} g(t-\delta t, x-\sqrt{\delta t})+@ \delta t \\
& \geq \frac{1}{2} \mu(t-\delta t, x+\sqrt{\delta t})+\frac{1}{2} g(t-\delta t, x-\sqrt{\delta t})+@ \delta t \\
& =\mu(t-2 \delta t, x)+@ \delta t,
\end{aligned}
$$

in contradiction with the supoosed maximality of $\mu$. Hence $t \lesssim T$. We conclude that $(t, x) \in S$ for $t \simeq T$ and $x \gtrsim 0$. Then $\phi(t) \simeq 0$ for $t \simeq T$ because $\phi$ is $D$-nondecreasing, which means that $\phi$ is $S$-continuous infinitely close to the horizon.

Lemma 23 states that the solution stays equal to the prescribed function infinitely close to the limited part of the horizon; as such it expresses $S$-continuity in time, which will be needed in Subsection 7.3. It gives also a growth condition at infinity. The lemma follows directly from Lemma 15 .

Lemma 23. One has $u(t, x) \simeq g(T, x)$ for limited $x \geq \phi(t)$ and $u(t, x) \leq 2 K \exp (2 C x)$ for all $x \geq \phi(t)$.

Proof of Theorem 19. Assume $0 \leq t<T$ and $\phi(t)$ is limited. It follows from Lemma 23 that $u(t, y)$ is limited for $y>\phi(t)$, with at most standard exponential growth at infinity. Then, working with $t$ instead of $T$, we may prove along the lines of the proof of Lemma 22 that it is impossible to have $s<t, s \simeq t$ and $\phi(s) \gtrsim \phi(t)$. Then $\phi(s) \simeq \phi(t)$ because $\phi$ is $D$-nondecreasing. Hence $\phi$ is $S$-continuous at $t$. By the Lemma of the Local Shadow of [6, p. 99] the free boundary $\phi$ is $S$-continuous at least down to some $t_{0}$ with $t_{0} \succsim T$.

For convenience we will from now on assume a fifth condition. By Theorem 19 it does not restrict generality, for one could consider the free boundary only for times $t$ with $t_{0} \leq t \leq T$, with $t_{0}$ as given by the above proof.

H5. The free boundary is $S$-continuous at all times.

\section{2. $S$-continuity of the solution in space}

\section{Theorem 24. The solution is of class $S^{0}$ in space.}

Proof. Let $(t, x),(t, x+\epsilon) \in D$ and $\epsilon>0, \epsilon \simeq 0$. We need only to consider the case where $x \geq \phi(t)$ is limited. Here $u$ is clearly limited. If $t \simeq T$, by Lemma 23,

$$
u(t, x+\epsilon) \simeq g(T, x+\epsilon) \simeq g(T, x) \simeq u(t, x) .
$$


Let $t \lesssim T$. One has $g(\tau, \phi(\tau)+\epsilon) \simeq g(\tau, \phi(\tau))$ for all $\tau$ with $t \leq \tau \leq T$ and $g(T, y+\epsilon) \simeq$ $g(T, y)$ for all limited $y \geq 0$, with standard exponential growth at infinity. Then by (20) and Proposition 14.2

$$
u(t, x+\epsilon)-u(t, x) \gtrsim 0 .
$$

Also, by (21) and Proposition 14.2

$$
u(t, x+\epsilon)-u(t, x) \lesssim 0 .
$$

Combining, we obtain that $u(t, x+\epsilon) \simeq u(t, x)$. Hence $u$ is $S$-continuous in $x$.

Next proposition is a corollary.

Proposition 25. Let $(t, x) \in D$ with $x \simeq \phi(t)$. Then $u(t, x) \simeq g(t, x)$.

Proof. One has $u(t, x)=u(t, \phi(t)=g(t, \phi(t) \simeq g(t, x)$.

\section{3. $S$-continuity of the solution in time}

Theorem 26. The solution is of class $S^{0}$ in time.

The theorem follows from Lemma 23 and Proposition 27 below. Lemma 23 implies that the solution is $S$-continuous in time infinitely close to the horizon. $S$-continuity in time infinitely close to the free boundary follows from the first part of the proof of Proposition 27 below. The second part of the proof deals with $S$-continuity at points away both from the horizon and the free boundary.

Proposition 27. Let $(t, x) \in D$ with $t \gtrsim T$, and $\epsilon \in \mathbb{T}, \epsilon>0, \epsilon \simeq 0, \epsilon / \delta t$ even. Let $x \geq \phi(t+\epsilon)$ be limited. Then

$$
u(t+\epsilon, x) \simeq u(t, x) .
$$

Proof. (i) $x \simeq \phi(t)$. Then also $x \simeq \phi(t+\epsilon)$, hence by Proposition 25

$$
u(t+\epsilon, x) \simeq g(t+\epsilon, \phi(t+\epsilon)) \simeq g(t, \phi(t)) \simeq u(t, x) .
$$

(ii) $x \gtrsim \phi(t)$. Then also $x \gtrsim \phi(t+\epsilon)$. By (34) and Lemma 23, for all $\tau \geq t+\epsilon$

$$
u(\tau, \phi(\tau))-u_{-\epsilon, 0}(\tau, \phi(\tau))=u(\tau, \phi(\tau))-u(\tau-\epsilon, \phi(\tau)) \simeq 0 .
$$

Hence $S_{\phi}\left(t+\epsilon, x ; u-u_{-\epsilon, 0}\right) \simeq 0$. Also, we have $u(T, x)-u_{-\epsilon, 0}(T, x)=u(T, x)-$ $u(T-\epsilon, x)$. Applying Lemma 23 again, we find that $u(T, x)-u_{-\epsilon, 0}(T, x) \simeq 0$ for limited $x$, while it has standard exponential growth for unlimited $x$. Then by formula (24) and Proposition 14.2,

$$
u(t+\epsilon, x)-u(t, x)=S_{\phi}\left(t+\epsilon, x ; u-u_{-\epsilon, 0}\right)+S_{T}\left(t+\epsilon, x ; u-u_{-\epsilon, 0}\right) \simeq 0 .
$$

Combining, we conclude that $u(t+\epsilon, x) \simeq u(t, x)$ holds for all limited $x$. 


\section{Higher order $S$-derivability of the solutions}

Theorem 28. The solution is of class $S^{n}$ for all standard $n \in \mathbb{N}$ at all $(t, x) \in D$ with $t \succsim T$ and $x \gtrsim \phi(t)$.

To prove the theorem it suffices to prove that, when $t \gtrsim T$ and $x \gtrsim \phi(t)$, the $n^{t h}$ discrete derivative $u_{0, n}$ of the solution with respect to space is limited for all standard $n \in \mathbb{N}$. Indeed, then $u_{n, 0}$ is also limited for $t \succsim T$ and $x \gtrsim \phi(t)$, because $u_{n, 0}(t-$ $2 n \delta t, x)=-\frac{1}{2} u_{0,2 n}(t, x-n \delta x)$. It follows that $u_{n, 0}$ and $u_{0, n}$ are $S$-continuous in both variables, for $u_{n+1,0}$ and $u_{0, n+1}$ are limited.

To estimate $u_{0, n}$ we write it in the form of a path sum with respect to an appropriate broken line. This broken line consists of a horizontal part $\eta \equiv\left(t, x^{\prime}\right)\left(t^{\prime}, x^{\prime}\right)$ below the point $(t, x)$ at appreciable distance $\left(x^{\prime} \succsim x\right)$, followed by a vertical part $\left(t^{\prime}, x^{\prime}\right)\left(t^{\prime}, x+\right.$ $\left.\frac{t^{\prime}-t}{\sqrt{\delta t}}\right)$ to the right of $(t, x)$ also at appreciable distance, i.e $t \lesssim t^{\prime}$; the vertical part goes upwards until hitting the upmost trajectory of the Wiener walk passing through $(t, x)$. The angular point $\left(t^{\prime}, x^{\prime}\right)$ is chosen to avoid the curve $\phi$; this is possible because $\phi$ is $S$-continuous. On behalf of (13) the solution satisfies

$$
u(t, x)=S_{\eta}(t, x ; u)+S_{t^{\prime}}(t, x ; u) .
$$

Then by Proposition 3 and 2

$$
\begin{aligned}
u(t, x)= & \sum_{\substack{t+\left(x^{\prime}-x\right) \sqrt{\delta t} \leq \tau \leq t^{\prime}\\
}} u\left(\tau, x^{\prime}\right) b_{2}\left(t-\tau-\delta t, x-x^{\prime}-\sqrt{\delta t}\right)+ \\
& \sum_{x^{\prime}<y<x+\frac{t^{\prime}-t}{\sqrt{\delta t}}} u\left(t^{\prime}, y\right)\left(b\left(t-t^{\prime}, y-\left(x-x^{\prime}\right)\right)-b\left(t-t^{\prime}, y+\left(x-x^{\prime}\right)\right)\right) .
\end{aligned}
$$

Next proposition extends (35) to differences with respect to space of the solution. It has a straightforward combinatorial proof.

Proposition 29. Let $(t, x) \in D$ with $t \gtrsim T$ and $x \gtrsim \phi(t)$. Then there exists $\left(t^{\prime}, x^{\prime}\right) \in D$ with $x^{\prime} \succsim x, t \succsim t^{\prime} \succsim T$ and $x^{\prime} \gtrsim \phi(\tau)$ for all $\tau \in\left[0 \cdots t^{\prime}\right]$, such that for all standard $n \in \mathbb{N}$

$$
\begin{aligned}
= & \sum_{t+\left(x^{\prime}-x\right) \sqrt{\delta t} \leq \tau \leq t^{\prime}}^{u_{0, n}(t, x)} u\left(\tau, x^{\prime}\right) b_{0, n+1}\left(t-\tau-\delta t, x-x^{\prime}-\sqrt{\delta t}\right) \delta x+ \\
& \sum_{x^{\prime}<y<x+\frac{t^{\prime}-t}{\sqrt{\delta t}}} u\left(t^{\prime}, y\right)\left(b_{0, n}\left(t-t^{\prime}, y-\left(x-x^{\prime}\right)\right)-b_{0, n}\left(t-t^{\prime}, y+\left(x-x^{\prime}\right)\right)\right) \delta x .
\end{aligned}
$$


In proving that $u_{0, n}$ is limited, the terms at times $\tau \simeq t$ in the first sum of (36) must be handled with care, for we are considering the difference quotients $b_{0, n+1}$ at infinitesimal times. We will prove that the sums are nearly equal to standard convergent improper Riemann-integrals, hence are limited. The second sum (36) is more easy to deal with.

Lemma 30. Let $(t, x) \in D$. Assume $0 \succsim x-x^{\prime} \succsim \infty$ and $t \lesssim t^{\prime} \succsim T$. Then for all standard $n \in \mathbb{N}$

$$
\begin{aligned}
& \sum_{x^{\prime}<y<x+\frac{t^{\prime}-t}{\delta t}} u\left(t^{\prime}, y\right)\left(b_{0, n}\left(t-t^{\prime}, y-\left(x-x^{\prime}\right)\right)-b_{0, n}\left(t-t^{\prime}, y+\left(x-x^{\prime}\right)\right)\right) \delta x \\
\simeq & \int_{x^{\prime}}^{\infty}{ }^{\circ} u\left(t^{\prime}, y\right)\left(G_{2}^{(n)}\left(t-t^{\prime}, y-\left(x-x^{\prime}\right)\right)-G_{2}^{(n)}\left(t-t^{\prime}, y+\left(x-x^{\prime}\right)\right)\right) d y .
\end{aligned}
$$

Proof. Notice that both $t-t^{\prime}$ and $x-x^{\prime}$ are appreciable. Hence by the higher-order DeMoivre-Laplace Theorem $b_{0, n}\left(t-t^{\prime}, y-\left(x-x^{\prime}\right)\right) \simeq G_{2}^{(n)}\left(t-t^{\prime}, y-\left(x-x^{\prime}\right)\right)$ and $b_{0, n}\left(t-t^{\prime}, y+\left(x-x^{\prime}\right)\right) \simeq G_{2}^{(n)}\left(t-t^{\prime}, y+\left(x-x^{\prime}\right)\right)$ for all limited $y>x^{\prime}$. Moreover $b_{0, n}\left(t-t^{\prime}, y-\left(x-x^{\prime}\right)\right), b_{0, n}\left(t-t^{\prime}, y-\left(x-x^{\prime}\right)\right)=£ \exp \left(-@ y^{2}\right)$ for all unlimited $y \in\left[x^{\prime} \cdot \cdot x+\frac{t^{\prime}-t}{\delta t}\right]$. Now $u\left(t^{\prime}, y\right)$ is $S$-continuous for all limited $y>x^{\prime}$, and by Proposition 15 has at most linear exponential growth for $y \in\left[x^{\prime} \cdot \cdot x+\frac{t^{\prime}-t}{\delta t}\right]$. So we have by the Lemma of Dominated Approximation

$$
\begin{aligned}
& \sum_{x^{\prime}<y<x+\frac{t^{\prime}-t}{\delta t}} u\left(t^{\prime}, y\right)\left(b_{0, n}\left(t-t^{\prime}, y-\left(x-x^{\prime}\right)\right)-b_{0, n}\left(t-t^{\prime}, y+\left(x-x^{\prime}\right)\right)\right) \delta x \\
& \simeq \int_{x^{\prime}}^{\infty}{ }^{\circ} u\left(t^{\prime}, y\right)\left(G_{2}^{(n)}\left(t-t^{\prime}, y-\left(x-x^{\prime}\right)\right)-G_{2}^{(n)}\left(t-t^{\prime}, y+\left(x-x^{\prime}\right)\right)\right) d y .
\end{aligned}
$$

Next lemma states that a similar result holds for the first sum.

Lemma 31. Let $(t, x) \in D$, and $n \in \mathbb{N}$ be standard. Assume $0 \gtrsim x-x^{\prime} \succsim \infty$ and $t \succsim t^{\prime} \succsim T$. Then

$$
\begin{aligned}
& \sum_{\left(x^{\prime}-x\right) \sqrt{\delta t} \leq \tau \leq t^{\prime}} u\left(\tau, x^{\prime}\right) b_{0, n+1}\left(\tau-t-\delta t, x-x^{\prime}-\sqrt{\delta t}\right) \delta x \\
\simeq & \int_{0}^{t^{\prime}}{ }^{\circ} u\left(\tau, x^{\prime}\right) G_{2}^{(n+1)}\left(\tau-t, x-x^{\prime}\right) d \tau .
\end{aligned}
$$

The lemma is a direct consequence of the next two lemmas, where the interval of summation respectively integration are divided into two parts: an appropriate interval of infinitesimal length near the origin and its complement with respect to the whole timeinterval. We start with the latter. 
Lemma 32. Let $(t, x) \in D$, and $n \in \mathbb{N}$ be standard. Assume $0 \gtreqless x-x^{\prime} \succsim \infty$ and $t \lesssim t^{\prime} \succsim T$. Then there exists $s>0, s \simeq 0$ such that

$$
\begin{aligned}
\sum_{s \leq \tau \leq t^{\prime}} u\left(\tau, x^{\prime}\right) b_{0, n+1}\left(\tau-t-\delta t, x-x^{\prime}\right. & -\sqrt{\delta t}) \delta x \\
& \simeq \int_{s}^{t^{\prime}}{ }^{\circ} u\left(\tau, x^{\prime}\right)\left(G_{2}^{(n+1)}\left(\tau-t, x-x^{\prime}\right) d \tau .\right.
\end{aligned}
$$

Proof. Let $s \gtrsim 0$. Then by the higher-order DeMoivre-Laplace Theorem for all $\tau \in\left[s \cdot t^{\prime}\right]$

$$
\begin{aligned}
b_{0, n+1}\left(\tau-t-\delta t, x-x^{\prime}-\sqrt{\delta t}\right) & \simeq G_{2}^{(n+1)}\left(\tau-t-\delta t, x-x^{\prime}-\sqrt{\delta t}\right) \\
& \simeq G_{2}^{(n+1)}\left(\tau-t, x-x^{\prime}\right) .
\end{aligned}
$$

Because $u\left(\tau, x^{\prime}\right)$ is limited for all $\tau \in\left[t \cdot t^{\prime}\right]$,

$$
\begin{aligned}
& \sum_{s \leq \tau \leq t^{\prime}} u\left(\tau, x^{\prime}\right) b_{0, n+1}\left(\tau-t-\delta t, x-x^{\prime}-\sqrt{\delta t}\right) \delta x \\
\simeq & \int_{s}^{t^{\prime}}{ }^{\circ} u\left(\tau, x^{\prime}\right)\left(G_{2}^{(n+1)}\left(\tau-t, x-x^{\prime}\right) d \tau .\right.
\end{aligned}
$$

By the Fehrele Principle (37) still holds for some $s \simeq 0$.

Lemma 33. Let $(t, x) \in D$, and $n \in \mathbb{N}$ be standard. Assume $0 \succsim x-x^{\prime} \succsim \infty$ and $t \succsim T$. Let $s>0, s \simeq 0$. Then

$$
\begin{aligned}
\sum_{0 \leq \tau \leq s} u\left(\tau, x^{\prime}\right) b_{0, n+1}(\tau-t-\delta t, x & \left.-x^{\prime}-\sqrt{\delta t}\right) \delta x \\
& \simeq \int_{0}^{s}{ }^{\circ} u\left(\tau, x^{\prime}\right) G_{2}^{(n+1)}\left(\tau-t, x-x^{\prime}\right) d \tau \simeq 0 .
\end{aligned}
$$

Proof. Let $l$ be limited such that $u\left(\tau, x^{\prime}\right) \simeq l$ for all $\tau \in[0 \cdots s]$. Since $\left(x-x^{\prime}\right) / \sqrt{\delta t}$ is unlimited, it follows from Lemma 8 that $b_{0, n+1}\left(\tau-t-\delta t, x-x^{\prime}-\sqrt{\delta t}\right) \simeq 0$ for all $\tau \in\left[\left(x-x^{\prime}\right) / \sqrt{\delta t} \cdot s\right]$. Hence

$$
\sum_{\left(x-x^{\prime}\right) / \sqrt{\delta t} \leq \tau \leq s} u\left(\tau, x^{\prime}\right) b_{0, n+1}\left(\tau-t-\delta t, x-x^{\prime}-\sqrt{\delta t}\right) \delta x \simeq 0 .
$$

Now $\int_{0}^{t^{\prime} \circ} u\left(\tau, x^{\prime}\right) G_{2}^{(n+1)}\left(\tau-t, x-x^{\prime}\right) d \tau$ is a standard convergent improper Riemannintegral. So $\int_{0}^{s} \circ u\left(\tau, x^{\prime}\right) G_{2}^{(n+1)}\left(\tau-t, x-x^{\prime}\right) d \tau \simeq 0$. Combining, we derive the lemma. 
Proof of Theorem 28: It follows directly from Proposition 29, Lemma 31 and Lemma 30 that there exists $\left(t^{\prime}, x^{\prime}\right) \in D$ with $0 \gtrsim x-x^{\prime} \gtrsim \infty, t \gtrsim t^{\prime} \gtrsim T$ and $x^{\prime} \gtrsim \phi(\tau)$ for all $\tau \in\left[0 \cdot t^{\prime}\right]$, such that for all standard $n \in \mathbb{N}$

$$
\begin{aligned}
& u_{0, n}(t, x) \simeq \int_{0}^{t^{\prime}}{ }^{\circ} u\left(\tau, x^{\prime}\right) G_{2}^{(n+1)}\left(\tau-t, x-x^{\prime}\right) d \tau+ \\
& \quad \int_{x^{\prime}}^{\infty}{ }^{\circ} u\left(t^{\prime}, y\right)\left(G_{2}^{(n)}\left(t-t^{\prime}, y-\left(x-x^{\prime}\right)\right)-G_{2}^{(n)}\left(t-t^{\prime}, y+\left(x-x^{\prime}\right)\right)\right) d y
\end{aligned}
$$

Because ${ }^{\circ} u$ and $G_{2}^{(n+1)}$ are standard and continuous

$$
\int_{0}^{t^{\prime}}{ }^{\circ} u\left(\tau, x^{\prime}\right) G_{2}^{(n+1)}\left(\tau-t, x-x^{\prime}\right) d \tau \simeq \int_{0}^{\circ t^{\prime}}{ }^{\circ} u\left(\tau,{ }^{\circ} x^{\prime}\right) G_{2}^{(n+1)}\left(\tau-{ }^{\circ} t,{ }^{\circ}\left(x-x^{\prime}\right)\right) d \tau .
$$

Applying the Lemma of Dominated Approximation, we find

$$
\begin{aligned}
& \int_{x^{\prime}}^{\infty}{ }^{\circ} u\left(t^{\prime}, y\right)\left(G_{2}^{(n)}\left(t-t^{\prime}, y-\left(x-x^{\prime}\right)\right)-G_{2}^{(n)}\left(t-t^{\prime}, y+\left(x-x^{\prime}\right)\right)\right) d y \\
\simeq & \int_{x^{\prime}}^{\infty}{ }^{\circ} u\left({ }^{\circ} t^{\prime}, y\right)\left(G_{2}^{(n)}\left(t-{ }^{\circ} t^{\prime}, y-{ }^{\circ}\left(x-x^{\prime}\right)\right)-G_{2}^{(n)}\left(t-{ }^{\circ} t^{\prime}, y+{ }^{\circ}\left(x-x^{\prime}\right)\right)\right) d y .
\end{aligned}
$$

Hence

$$
\begin{aligned}
u_{0, n}(t, x) & \simeq \int_{0}^{\circ{ }^{\prime}{ }^{\circ}} u\left(\tau,{ }^{\circ} x^{\prime}\right) G_{2}^{(n+1)}\left(\tau-{ }^{\circ} t,{ }^{\circ}\left(x-x^{\prime}\right)\right) d \tau+ \\
& \int_{x^{\prime}}^{\infty}{ }^{\circ} u\left(t^{\prime}, y\right)\left(G_{2}^{(n)}\left(t-t^{\prime}, y-\left(x-x^{\prime}\right)\right)-G_{2}^{(n)}\left(t-t^{\prime}, y+{ }^{\circ}\left(x-x^{\prime}\right)\right)\right) d y .
\end{aligned}
$$

We conclude that $u_{0, n}$, being infinitely close to a standard number, is limited. Hence $u$ is of class $S^{n}$ in $x$. Because $u_{n, 0}(t, x)=\frac{1}{2} u_{0,2 n}(t, x-n \delta x)$ the function $u$ is also of class $S^{n}$ in $t$. Hence $u$ is of class $S^{n}$.

\section{9. $S$-derivability of the solution infinitely close to the free boundary}

\section{1. $S$-derivability of the solution in space}

We prove the following theorem.

Theorem 34. The solution is of class $S^{1}$ in the space variable on $D$, in particular $u_{0,1}(t, x) \simeq g_{0,1}(t, \phi(t))$ for $x \simeq \phi(t), x \geq \phi(t)$ and $u_{0,1}(t, x) \simeq g_{0,1}(T, x)$ for $t \simeq T$. 
We start with the case $t \simeq T$. Then we derive that the solution is $S$-differentiable in the space-variable exactly at the free boundary, with discrete derivative nearly equal to the discrete derivative with respect to the space-variable of the function $g$ at the boundary. The latter discrete derivative is used to express the discrete derivative of the solution with respect to the space-variable at points above the boundary as a path sum, up to an infinitesimal. Informally, for points $(t, x)$ infinitely close to the boundary, the paths almost surely hit the boundary within infinitesimal time, thus using values of $g_{0,1}(\tau, \phi(\tau))$ for $\tau \simeq t$, which are infinitely close to $g_{0,1}(t, \phi(t))$. So $u_{0,1}(t, x) \simeq g_{0,1}(t, \phi(t))$ indeed (Lemma 37). A similar argument holds near the horizon.

Proposition 35. Let $(t, x) \in D$ with $t \simeq T, x \geq \phi(t)$. Then $u_{0,1}(t, x) \simeq g_{0,1}(T, x)$.

Proof. Observe that $g_{0,1}$ is of class $S^{0}$ in $x$ at the horizon $T$, with standard linear exponential growth. By (20)

$$
u_{0,1}(t, x) \geq S_{\phi}\left(t, x ; g_{0,1}\right)+S_{T}\left(t, x ; g_{0,1}\right) .
$$

Then $u_{0,1}(t, x) \gtrsim g_{0,1}(T, x)$ by Proposition 15.1. By (21)

$$
\begin{aligned}
u_{0,1}(t, x) \leq & \left.\sum_{t \leq \tau \leq T} P_{\phi-\delta x}(t, x) ; \tau, \phi(\tau)-\delta x\right) g_{0,1}(\tau, \phi(\tau)-\delta x) \\
& \left.+\sum_{\phi(T)-\delta x<y} P_{\phi-\delta x}(t, x) ; T, y\right) g_{0,1}(T, y-\delta x)
\end{aligned}
$$

Applying again Proposition 15 we find that $u_{0,1}(t, x) \lesssim g_{0,1}(T, x-\delta x) \simeq g_{0,1}(T, x)$. We conclude that $u_{0,1}(t, x) \simeq u_{0,1}(T, x)$.

Proposition 36. Let $0 \leq t \lesssim T$. Then $u_{0,1}(t, \phi(t)) \simeq g_{0,1}(t, \phi(t))$.

Proof. One has $u(t, \phi(t)+\delta x) \geq g(t, \phi(t)+\delta x)$, so $u_{0,1}(t, \phi(t)) \geq g_{0,1}(t, \phi(t))$. On the other hand, by (21) and Proposition 14.1

$$
\begin{aligned}
& u_{0,1}(t, \phi(t)) \leq \sum_{t \leq \tau \leq T} P_{\phi-\delta x}(t, \phi(t) ; \tau, \phi(\tau)-\delta x) g_{0,1}(\tau, \phi(\tau)-\delta x) \\
& +\sum_{\phi(T)-\delta x<y} P_{\phi-\delta x}(t, \phi(t) ; T, y) g_{0,1}(T, y-\delta x) \\
\simeq & g_{0,1}(t, \phi(t)-\delta x) \simeq g_{0,1}(t, \phi(t)) .
\end{aligned}
$$

Combining, we derive that $u_{2}(t, \phi(t)) \simeq g_{0,1}(t, \phi(t))$.

Proposition 37. Let $(t, x) \in D$ with $t \nRightarrow T$ and $x \geq \phi(t), x \simeq \phi(t)$. Then $u_{0,1}(t, x) \simeq$ $g_{0,1}(t, \phi(t))$. 
Proof. By Proposition 14.1 and 36

$$
u_{0,1}(t, x)=S_{\phi}\left(t, x ; u_{0,1}\right)+S_{T}\left(t, x ; g_{0,1}\right) \simeq u_{0,1}(t, \phi(t)) \simeq g_{0,1}(t, \phi(t)) .
$$

Proof of Theorem 34. For $(t, x) \in D$ with $t \gtrsim T$ and $x \gtrsim \phi(t)$ the property of being of class $S^{1}$ in $x$ is a special case of Theorem 28. If $t \simeq T$ and $x \geq \phi(t)$ the conclusion follows from Proposition 35, and for $t \gtrsim T$ and $x \simeq \phi(t)$ the conclusion follows from Proposition 37, noting that $g_{0,1}$ is of class $S^{0}$.

\section{2. $S$-derivability of the solution in time}

Consider a point $(t, \phi(t))$ on the free boundary with $t \lesssim T$, such that the solution at the previous point in time, at $(t-2 \delta t, \phi(t))$, is not on the free boundary, i.e. of the form $u(t-2 \delta t, \phi(t))=\mu(t-2 \delta t, \phi(t))$. We derive that the solution is $S$-differentiable in the time-variable at this point, with discrete derivative nearly equal to the discrete derivative with respect to the space-variable of the function $g$ at the boundary. As in the previous subsection, the latter discrete derivative is used to express the discrete derivative of the solution with respect to the time-variable at points above the boundary as a path sum, up to an infinitesimal. For the points $(t, x)$ infinitely close to the boundary, the proof that $u_{1,0}(t, x) \simeq g_{1,0}(t, \phi(t))$ uses also the same argument as in the previous section: the paths almost surely hit the boundary within infinitesimal time, so essentially only values close to $g_{1,0}(t, \phi(t))$ matter. In contrast to the discrete derivative with respect to space, the discrete derivative with respect to time needs not to be limited close to the horizon and the free boundary, since $g$ takes steps of order $\sqrt{\delta t}$ instead of $\delta t$. In fact, we will prove the following theorem.

Theorem 38. Let $(t, x) \in D$ be such that $2 \delta t \leq t \lesssim T$ and $x>\phi(t), x \simeq \phi(t)$. Then $u_{1,0}(t, x) \simeq g_{1,0}(t, \phi(t))$.

We consider first the point where $\phi$ takes a downward step. We will present the somewhat intricate calculations with some detail.

Lemma 39. Let $2 \delta t \leq t \lesssim T$. If $u(t-2 \delta t, \phi(t))=\mu(t-2 \delta t, \phi(t))$,

$$
u_{1,0}(t-2 \delta t, \phi(t)) \simeq g_{1,0}(t-2 \delta t, \phi(t)) .
$$

Moreover,

$$
\left.\sum_{y>\phi(T-2 \delta t)} P(t, \phi(t) ; T, y)\right) u_{1,0}(T-2 \delta t, y) \simeq 0 .
$$

Proof. Because $u(t, \phi(t))=g(t, \phi(t))$ and $u(t-2 \delta t, \phi(t))>g(t-2 \delta t, \phi(t))$,

$$
u_{1,0}(t-2 \delta t, \phi(t))<g_{1,0}(t-2 \delta t, \phi(t)) .
$$


To prove that $u_{1,0}(t-2 \delta t, \phi(t)) \gtrsim g_{1,0}(t-2 \delta t, \phi(t))$, we note that by (27)

$$
\begin{aligned}
u(t, \phi(t))-u(t-2 \delta t, \phi(t)) \geq & S_{\phi}\left(t-2 \delta t, \phi(t) ; g-g_{-2 \delta t, 0}\right) \\
& +S_{T-2 \delta t}\left(t-2 \delta t, \phi(t) ; u_{2 \delta t, 0}-u\right) .
\end{aligned}
$$

The first possible hit of the curve $\phi$ made by trajectories of the Wiener walk starting at $(t-2 \delta t, \phi(t))$ is at $(t, \phi(t)$, and almost all trajectories will hit $\phi$ infinitely close to $(t, \phi(t)$. Then by Proposition 14.1, the first sum of the right-hand side of (41) is nearly equal to $g_{1,0}(t, \phi(t)) \simeq g_{1,0}(t-2 \delta t, \phi(t))$. Since $u_{2 \delta t, 0}(T-2 \delta t, y)=u(T, y)=g(T, y)$, the second sum of (41) amounts to

$$
\begin{aligned}
& S_{T-2 \delta t}\left(t-2 \delta t, \phi(t) ; u_{2 \delta t, 0}-u\right) \\
= & \left.\sum_{y>\phi(T-2 \delta t)} P(t, \phi(t) ; T-2 \delta t, y)\right) \frac{(g(T, y)-u(T-2 \delta t, y))}{2 \delta t} .
\end{aligned}
$$

We will prove that this sum is infinitesimal. We start by examining the term at $(T, 0)$. If $u(T-2 \delta t, 0)=g(T-2 \delta t, 0)$,

$$
\frac{(g(T, 0)-u(T-2 \delta t, 0))}{2 \delta t}=g_{1,0}(T-2 \delta t, 0),
$$

which is limited, because $g_{1}^{\prime}(T, 0)$ is well-defined. Also the left-derivative with respect to space at $(T, 0)$ is well-defined, so we may use a Taylor-expansion at $(T, 0)$ to obtain

$$
\begin{aligned}
& \frac{(g(T, 0)-u(T-2 \delta t, 0)}{2 \delta t} \\
= & \frac{g(T, 0)-\left(\frac{1}{2} g(T-\delta t,-\sqrt{\delta t})+\frac{1}{4} g(T, 0)+\frac{1}{4} g(T, \delta x)\right)}{2 \delta t} \\
= & \frac{\delta t\left(\frac{1}{2} g_{1}^{\prime}(T, 0)+\oslash\right)-\frac{1}{2} \sqrt{\delta t}\left(g_{2}^{\prime}(T, 0)+\oslash\right)+\frac{1}{4} \delta x\left(g_{2}^{\prime}(T, 0)+\oslash\right)}{2 \delta t} \\
= & \frac{\oslash}{\sqrt{\delta t}} .
\end{aligned}
$$

Now the probability of transition $P(t, \phi(t) ; T-2 \delta t, 0)$ is bounded by the probability of a trajectory of the Wiener walk going from $(t, \phi(t)$ to $(T-2 \delta t, 0)$ without restrictions, i.e. by $b(T-t+2 \delta t, \phi(t)) \delta x=£ \delta x$. Hence in both cases

For $y>0$

$$
P(t, \phi(t) ; T-2 \delta t, 0) \frac{(g(T, 0)-u(T-2 \delta t, 0)}{2 \delta t} \simeq 0 .
$$

$$
\begin{aligned}
\frac{(g(T, y)-u(T-2 \delta t, y))}{2 \delta t} & =u_{1,0}(T-2 \delta t, y)=u_{0,2}(T, y-\delta x) \\
& =g_{0,2}(T, y-\delta x),
\end{aligned}
$$


which has at most standard exponential growth. Then (42) and Proposition 13 imply that $S_{T-2 \delta t}\left(t-2 \delta t, \phi(t) ; u_{2 \delta t, 0}-u\right) \simeq 0$. Hence $u_{1,0}(t-2 \delta t, \phi(t)) \gtrsim g_{1,0}(t-2 \delta t, \phi(t))$. Combining with (40), we derive (38).

REMARK. -

Formula (42) shows that in general, the solution is not of class $S^{1}$ in time close to the point where the free boundary is infinitely close to the horizon, for if $u(T-2 \delta t, 0)=$ $\mu(T-2 \delta t, 0)$, i.e, the free boundary takes two successive downward steps (counting backward $)$, the discrete derivative $(g(T, 0)-u(T-2 \delta t, 0) / 2 \delta t$ may be unlimited.

Proof of Theorem 38. By (24), at points of the form $(t-2 \delta t, x)$

$$
\left.u_{1,0}(t-2 \delta t, x)=S_{\phi}\left(t, x ;\left(u_{-2 \delta t, 0}\right)_{1,0}\right)+S_{T}\left(t, x ;\left(u_{-2 \delta t, 0}\right)_{1,0}\right)\right) .
$$

Then $u_{1,0}(t-2 \delta t, x) \simeq u_{1,0}(t-2 \delta t, \phi(t))$ by Lemma 14.1, while $u_{1,0}(t-2 \delta t, \phi(t)) \simeq$ $g_{1,0}(t-2 \delta t, \phi(t))$ by Lemma 39. This implies the theorem.

\subsection{Non $S$-derivability in $2^{\text {nd }}$ order of the solution in space}

The previous sections showed that the function $u$ is of class $S^{1,1}$, with the possible exception of points infinitely close to the horizon. Also, since the function $u$ is of class $S^{n}$ for any standard $n \in \mathbb{N}$ in the continuation region at points which are neither infinitely close to the horizon, nor the free boundary, in particular it is there of class $S^{1,2}$. Here we show that $u$ is not of class $S^{1,2}$ infinitely close to the free boundary. Intuitively this cannot be because $u$ satisfies the discrete heat equation in the continuation region, while in the stopping region $g$ does not.

Theorem 40. The second-order difference quotient $u_{0,2}$ is not $S$-continuous at points $(t, \phi(t))$ with $t \lesssim T$, in particular $u_{0,2}(t, \phi(t)-2 \delta x) \nRightarrow u_{0,2}(t, \phi(t)+\delta x)$.

Proof. Let $x \simeq y \simeq \phi(t)$ with $x+2 \delta x \leq \phi(t)<y$. By (33) and Theorem 38

$$
g_{0,2}(t, x) \succsim-2 g_{1,0}(t, x) \simeq-2 u_{1,0}(t, y)=u_{0,2}(t, y) .
$$

This implies the theorem.

\section{References}

[1] I.P. VAN DEN BERG, "Nonstandard asymptotic analysis", Springer Lect. Notes Math. vol. $1249,1987$.

[2] I.P. VAN DEN BERG, "On the relation between elementary partial difference equations and partial differential equations”, Ann. Pure Appl. Logic, vol. 92, 1998. 
[3] I.P. VAN DEN BERG, "Principles of infinitesimal stochastic and financial analysis", World Scientific, 2000.

[4] I.P. VAN DEN BERG, "Discretisations of higher order and the theorems of Faà di Bruno and DeMoivre-Laplace", Journal of Logic and Analysis, vol. 5, num. 6, 2013.

[5] N.J. Cutland, P.E. Kopp, W. Willinger, M.C. Wyman, "Convergence of Snell Envelopes and Critical Prices in the American Put", in Mathematics of Derivative Securities, M. Dempster, S. Pliska (eds.), Camb. Univ. Press, 1997.

[6] F. Diener, G. ReEB, “Analyse Non Standard”, Hermann, 1995.

[7] A. FRIEDMAN, "Parabolic variational inequalities in one space dimension and smoothness of the free boundary", Journal of Func. Anal., vol. 18, 1975.

[8] M. Gevrey, "Sur les équations aux dérivées partielles du type parabolique", Journal de Math. Pures et Appl., vol. 9, num. 6, 1913.

[9] D. LAMBERTON, "Critical price of an american option near maturity", in Seminar on stochastic analysis, M. Bolthausen, M. Dozzi, F. Russo (eds.), Birkhauser, Progress in Probability, vol. 36, 1995.

[10] D.P.J. LEISEN, "Pricing the american put option: a detailed convergence analysis for binomial models", Journal of Economic Dynamics and Control, vol. 22, 1998.

[11] P.L.J. VAn MoerbeKe, “An optimal stopping problem with linear reward”, Acta Mathematica, vol. 132, 1974.

[12] P.L.J. VAn MoERBeKe, "On optimal stopping and free boundary problems", Rational Mech. Anal., vol. 60, 1976.

[13] E. Nelson, "Radically Elementary Probability Theory", Princeton University Press, 1987. 